\title{
THE MAGNETIC FIELD AND POLARIZATION PROPERTIES OF RADIO GALAXIES IN DIFFERENT ACCRETION STATES
}

\author{
S. P. O’Sullivan ${ }^{1,2,3}$, B. M. Gaensler ${ }^{1,3,4}$, M. A. Lara-López ${ }^{2}$, S. van VelzeN ${ }^{5}$, J. K. Banfield ${ }^{6,7}$, and J. S. Farnes ${ }^{1,3}$ \\ ${ }^{1}$ Sydney Institute for Astronomy, School of Physics, The University of Sydney, NSW 2006, Australia; shane@astro.unam.mx \\ ${ }^{2}$ Instituto de Astronomía, Universidad Nacional Autónoma de México (UNAM), A.P. 70-264, 04510 México, D.F., Mexico \\ ${ }^{3}$ ARC Centre of Excellence for All-sky Astrophysics (CAASTRO), 44 Rosehill Street, Redfern, NSW 2016, Australia \\ ${ }^{4}$ Dunlap Institute for Astronomy and Astrophysics, The University of Toronto, 50 St. George Street, Toronto, ON M5S 3H4, Canada \\ ${ }^{5}$ Department of Physics and Astronomy, The Johns Hopkins University, Baltimore, MD 21218, USA \\ ${ }^{6}$ CSIRO Australia Telescope National Facility, P.O. Box 76, Epping, NSW, 1710, Australia \\ ${ }^{7}$ Research School of Astronomy and Astrophysics, Australian National University, Weston Creek, ACT 2611, Australia \\ Received 2015 January 27; accepted 2015 April 24; published 2015 June 9
}

\begin{abstract}
We use the integrated polarized radio emission at $1.4 \mathrm{GHz}\left(\Pi_{1.4 \mathrm{GHz}}\right)$ from a large sample of active galactic nuclei (AGN; 796 sources at redshifts $z<0.7)$ to study the large-scale magnetic field properties of radio galaxies in relation to the host galaxy accretion state. We find a fundamental difference in $\Pi_{1.4 \mathrm{GHz}}$ between radiative-mode AGN (i.e., high-excitation radio galaxies (HERGs) and radio-loud QSOs) and jet-mode AGN (i.e., low-excitation radio galaxies (LERGs)). While LERGs can achieve a wide range of $\Pi_{1.4 \mathrm{GHz}}$ (up to $\sim 30 \%$ ), the HERGs and radioloud QSOs are limited to $\Pi_{1.4 \mathrm{GHz}} \lesssim 15 \%$. A difference in $\Pi_{1.4 \mathrm{GHz}}$ is also seen when the sample is divided at $0.5 \%$ of the total Eddington-scaled accretion rate, where the weakly accreting sources can attain higher values of $\Pi_{1.4 \mathrm{GHz}}$. We do not find any clear evidence that this is driven by intrinsic magnetic field differences of the different radio morphological classes. Instead, we attribute the differences in $\Pi_{1.4 \mathrm{GHz}}$ to the local environments of the radio sources, in terms of both the ambient gas density and the magnetoionic properties of this gas. Thus, not only are different large-scale gaseous environments potentially responsible for the different accretion states of HERGs and LERGs, we argue that the large-scale magnetized environments may also be important for the formation of powerful AGN jets. Upcoming high angular resolution and broadband radio polarization surveys will provide the high-precision Faraday rotation measure and depolarization data required to robustly test this claim.
\end{abstract}

Key words: galaxies: active - galaxies: magnetic fields - radio continuum: galaxies

\section{INTRODUCTION}

The interaction of powerful relativistic jets in radio-loud active galactic nuclei (AGN) with their environment is observed to strongly affect the dynamics of interstellar and intergalactic gas (e.g., Bîrzan et al. 2008), likely impacting the formation and evolution of the most massive galaxies in the universe (e.g., Best et al. 2006; Croton et al. 2006). There exists a wide variety of radio-loud AGN jet types and powers, whose appearance is considered to be directly related to some combination of the intrinsic supermassive black hole properties (i.e., mass and spin), the amount of material feeding the black hole, the availability of large amounts of magnetic flux, and the properties of the environment into which the jet propagates (e.g., Stawarz et al. 2008; Laing \& Bridle 2014; Zamaninasab et al. 2014). One of the key open questions in the study of AGN is how the production of powerful relativistic jets in radio-loud AGN is related to the accretion system of the host galaxy. While substantial progress has been made in our understanding of the relation between the nuclear emission properties and the large-scale radio emission in AGN (e.g., Rawlings \& Saunders 1991; Laing et al. 1994; Hardcastle et al. 2007; Buttiglione et al. 2010; de Gasperin et al. 2011; van Velzen et al. 2013; Mingo et al. 2014), these studies have not yet considered the magnetic properties of the source or its environment, even though the magnetic field is well accepted as a key parameter in AGN physics (e.g., Meier 2002; Sikora \& Begelman 2013).

The host galaxies of radio-loud AGN display a large range of optical spectral signatures that can be grouped into at least two main modes (Hine \& Longair 1979; Laing et al. 1994): high- excitation radio galaxies (HERGs) and low-excitation radio galaxies (LERGs). The HERGs have strong, high-ionization emission lines and represent the classical picture of an AGN with an optically thick, geometrically thin, radiatively efficient accretion disk (Shakura \& Sunyaev 1973). This class represents the most optically luminous AGN (including radio-loud QSOs), a population that can be easily identified out to high redshift $(z>3)$. This class of AGN are also sometimes referred to as "radiative-mode," "quasar-mode," or "cold-mode" AGN. On the other hand, in the LERG class of objects, the optical emission lines are very weak or nonexistent, but the presence of radio jets clearly identifies the source as an AGN. In this case, the presence of a radiatively inefficient accretion flow is inferred (e.g., Narayan \& Yi 1995). LERGs are also known as "jet-mode," "radio-mode," or "hotmode" AGN. HERGs and LERGs have been proposed to be fuelled in fundamentally different ways (e.g., Best et al. 2005). The high radiative luminosities of HERGs are considered to be fuelled by a large supply of cold gas for accretion, potentially provided by a merger with a gas-rich disk galaxy or internal dynamical processes in the host galaxy itself. The radiatively inefficient, low-luminosity LERGs require much lower gas accretion rates, potentially provided by Bondi accretion of the hot X-ray halos surrounding their massive elliptical galaxy hosts (Hardcastle et al. 2007).

Jets from both HERGs and LERGs are generally considered to be launched in similar manners from the vicinity of the central supermassive black hole of their host galaxy by largescale, ordered magnetic fields (Blandford \& Znajek 1977; Blandford \& Payne 1982; Sikora \& Begelman 2013). These 
intrinsically magnetized jets can propagate to large ( $\gg \mathrm{kpc}$ ) distances, carrying energy and magnetic fields into their ambient galactic and extragalactic environments. However, as the jets propagate through their host galaxy, the radio emission structure changes, presumably in part due to the type of interaction with the ambient environment. In general, the largescale radio morphology is typically divided into two main types (Fanaroff \& Riley 1974): low radio power Fanaroff-Riley Class 1 (FR1) sources where the inner jet and core have the brightest radio emission, and high radio power FR2 sources where the brightest radio emission is located at the jet termination "hotspots." The traditional FR1/FR2 radio luminosity density divide occurs at $\sim 10^{25} \mathrm{~W} \mathrm{~Hz}^{-1}$ (Fanaroff \& Riley 1974), with a later study by Ledlow \& Owen (1996) showing that the FR1/FR2 divide also depends on the optical magnitude of the host galaxy, with the division occurring at higher radio luminosities in more optically luminous hosts. This result indicates that the FR1/FR2 morphology is most likely due to a combination of jet power and environment, since it is thought that the Ledlow \& Owen (1996) division arises due to jets being more easily disrupted into FR1 morphologies in the more massive host galaxies.

The FR1 and FR2 populations also appear to differ in their accretion states, with HERGs often associated with FR2s and LERGs mainly of the FR1 type (e.g., Laing et al. 1994). The common interpretation of this association is that with a higher accretion rate, due to the plentiful supply of cold gas, the HERGs can produce more powerful jets, which are more likely to develop into FR2-type sources, while the weakly accreting LERGs produce the less powerful FR1 jets. However, at low redshift, where both LERGs and HERGs can be equally well identified, there are several examples of FR1-HERGs and FR2LERGs (Hardcastle et al. 2007; Best 2009; Buttiglione et al. 2010). A detailed comparison of the FR type and host galaxy accretion mode found that the FR class was independent of the HERG/LERG divide (Gendre et al. 2013). Thus, the nature of the relationship between the accretion mode of the host galaxy, the formation of powerful radio jets, the morphological appearance of these jets on large scales, and the large-scale environment is still unclear.

The radio polarization properties of radio-loud AGN are important because they probe the internal structure of the jet magnetic field, as well as providing a unique diagnostic of the magnetoionic environment of the radio source through Faraday rotation of the linear polarization (e.g., Laing 1988; Garrington et al. 1988; Laing et al. 2008). The relationship between the large-scale polarization properties of the radio jets/lobes and the host galaxy accretion state is important not only because it is a sensitive probe of the local environment, and thus the origin of the gas fueling the black hole, but it also probes the magnetic field properties of the gas, and therefore helps provide an assessment of the ability of the black hole to accumulate the sufficiently large amounts of magnetic flux required for launching powerful radio-loud jets (e.g., Tchekhovskoy et al. 2012).

In this paper, we present an analysis of the accretion properties of host galaxies (as probed by nuclear optical emission lines) in relation to the large-scale magnetic field properties of their AGN jet (as probed by linearly polarized radio emission). In Section 2, we describe the construction of our sample of polarized HERG and LERG sources, the classification of their total intensity radio morphologies and the measurement of their linear sizes. In Section 3, we present our results on the integrated $1.4 \mathrm{GHz}$ polarization properties of HERGs and LERGs. We also investigate the dependence of the polarization on radio morphology, linear size, Faraday rotation, and the Eddington-scaled accretion rate. In Section 4, we discuss the implications of our results for the link between the large-scale magnetized environment of HERGs and LERGs and their black hole accretion state. Our conclusions are listed in Section 5. Throughout this paper, we assume a flat $\Lambda$ CDM cosmology with $H_{0}=67.3 \mathrm{~km} \mathrm{~s}^{-1} \mathrm{Mpc}^{-1}, \Omega_{M}=0.315$, and $\Omega_{\Lambda}=0.685$ (Planck Collaboration et al. 2014).

\section{SAMPLE CONSTRUCTION}

The only high-resolution radio survey covering a significant fraction of the sky $(\sim 82 \%)$ with polarization sensitivity is the NRAO VLA Sky Survey (NVSS), conducted at $1.4 \mathrm{GHz}$ (Condon et al. 1998). The NVSS has an angular resolution of $45^{\prime \prime}$ with an approximately uniform $\mathrm{rms}$ sensitivity of $\sim 0.45$ mJy beam ${ }^{-1}$ in Stokes $I$ and $\sim 0.29$ mJy beam $^{-1}$ in Stokes $Q$ and $U$. While the NVSS catalog already lists the basic integrated polarization properties, we have generated our own catalog by downloading the full NVSS IQU mosaics ${ }^{8}$ and using the source-finding program AEGEAN (Hancock et al. 2012). This was done as part of a larger project in order to better characterize the morphologies and multiple-component nature of polarized sources in the NVSS and has generated catalog positions for components in polarized intensity, Stokes $Q$ and Stokes $U$, independent of Stokes $I$. AEGEAN is an optimized source finder designed for reliably identifying compact radio sources based on a Laplacian kernel. It finds peaks in an image based on a "seed" threshold $\left(\sigma_{s}\right)$ and grows an island of pixels around that peak based on a "flood" threshold $\left(\sigma_{f}\right)$, where the local image noise level $\left(\sigma_{\text {rms }}\right)$ is estimated over a $20 \times 20$ beam area. A curvature image is then used to determine how many Gaussian components are used to describe the island, returning a catalog of component flux densities and angular sizes. For our search for polarization sources in the NVSS polarized intensity images $\left(p=\left(Q^{2}+U^{2}\right)^{1 / 2}\right)$, we used $\sigma_{f}=7 \sigma_{\mathrm{rms}}$ and $\sigma_{s}=8 \sigma_{\mathrm{rms}}$, with $\sigma_{\mathrm{rms}} \sim 0.2 \mathrm{mJy}$ being typical for most fields. This has resulted in the inclusion of only those polarized sources with peak polarized intensities above $8 \sigma$ of the local noise level in polarized intensity. Polarization bias was corrected for using the estimator $p_{0}=\left(p^{2}-\sigma_{Q U}^{2}\right)^{1 / 2}$, where $p_{0}$ is an estimate of the true polarized intensity (Simmons \& Stewart 1985). This has produced catalogs of $\sim 87,000$ components in polarized intensity and total intensity (Stokes $I$ ), as well as Stokes $Q$ and $U$ individually, over the entire NVSS area. A subset of that catalog is used here. The full polarization catalog will be presented elsewhere.

\subsection{HERG/LERG Parent Sample}

In order to calculate physically interesting quantities, we need to match each polarized radio source with its spectroscopically identified optical host galaxy. The largest optical spectroscopic survey overlapping with the NVSS is the Sloan Digital Sky Survey (SDSS), which covers $\sim 10,000$ square degrees of the Northern Hemisphere sky (DR 7; Abazajian et al. 2009). In addition to this, the Faint Images of the Radio Sky at Twenty centimetres (FIRST) survey (Becker

\footnotetext{
www.cv.nrao.edu/nvss/
} 
et al. 1995), which overlaps with the SDSS survey area, has a similar sensitivity limit as the NVSS but a significantly higher angular resolution of $5^{\prime \prime}$, which aids substantially in obtaining reliable cross-matches with the SDSS. Combining the NVSS, FIRST, and SDSS catalogs, Best \& Heckman (2012), hereafter BH12, produced a large sample of radio-loud AGN, with spectroscopic classification into HERGs and LERGs out to a redshift $z \sim 0.7$. They used the emission line information from the value-added spectroscopic catalogs available at http://www. mpa-garching.mpg.de/SDSS/ (cf. Brinchmann et al. 2004) to robustly classify 481 HERGs and 9863 LERGs out of 18,286 radio-loud AGN. We also use the line emission information from these catalogs in this paper.

\subsection{HERG/LERG Polarization Sample}

We constructed the sample used in this paper by crossmatching our NVSS polarization catalog with the BH12 catalog of HERGs and LERGs. Since BH12 have already carefully cross-matched the NVSS sources with their SDSS optical host galaxies using the additional, higher-resolution information provided by FIRST, our catalog is immediately highly reliable. Our initial catalog used a cross-matching radius of 1 arcmin and produced 802 matches. After manual visual inspection of the optical (SDSS) and radio (NVSS and FIRST) images of all matches, we found only six matches that had either misidentified optical hosts or were in confused fields (i.e., there was a potential contribution to $\Pi_{1.4 \mathrm{GHz}}$ by radio emission from an unrelated radio source). Thus, the final catalog has 796 polarized radio sources, the vast majority of which are unresolved in the NVSS ( $96 \%)$.

In the case of multiple NVSS components related to one radio source, we first integrated the contribution of Stokes $Q$ and $U$ from each component before calculation of the polarized intensity, and then divided by the sum of the total intensity components to get the integrated degree of polarization, $\Pi_{1.4 \mathrm{GHz}}$. The polarization bias correction was applied to the integrated polarized intensity. Ideally the bias correction should be applied to each component first, but that would have prevented us from comparing the polarization properties of the resolved sources with the unresolved sources in our analysis. In total, there are 32 resolved sources (in Stokes $I$ ) in our sample. Only three of these resolved sources have multiple polarized components, 19 have polarized component major axes greater than 45", while the remainder have compact polarized components (major axis $\leqslant 45^{\prime \prime}$ ). For the three multiplecomponent polarized sources, the higher noise level of $\sigma_{Q U}$ for the bias correction is calculated as the quadrature sum of the $\sigma_{Q}$ and $\sigma_{U}$ noise for each component.

Figure 1 shows examples of two SDSS galaxies (one HERG and one LERG, with their accompanying optical spectrum shown) overlaid with the corresponding NVSS $I$ and $p$ images, as well as the FIRST total intensity image. Note how the radio images appear almost identical but the optical spectra differ considerably.

\subsection{FIRST Morphological Analysis}

The high angular resolution FIRST images allow us to classify the total intensity morphology of all the polarized sources, and to calculate the projected linear size from the total angular extent of the FIRST total intensity emission. We manually classified the radio morphology of the 796 polarized
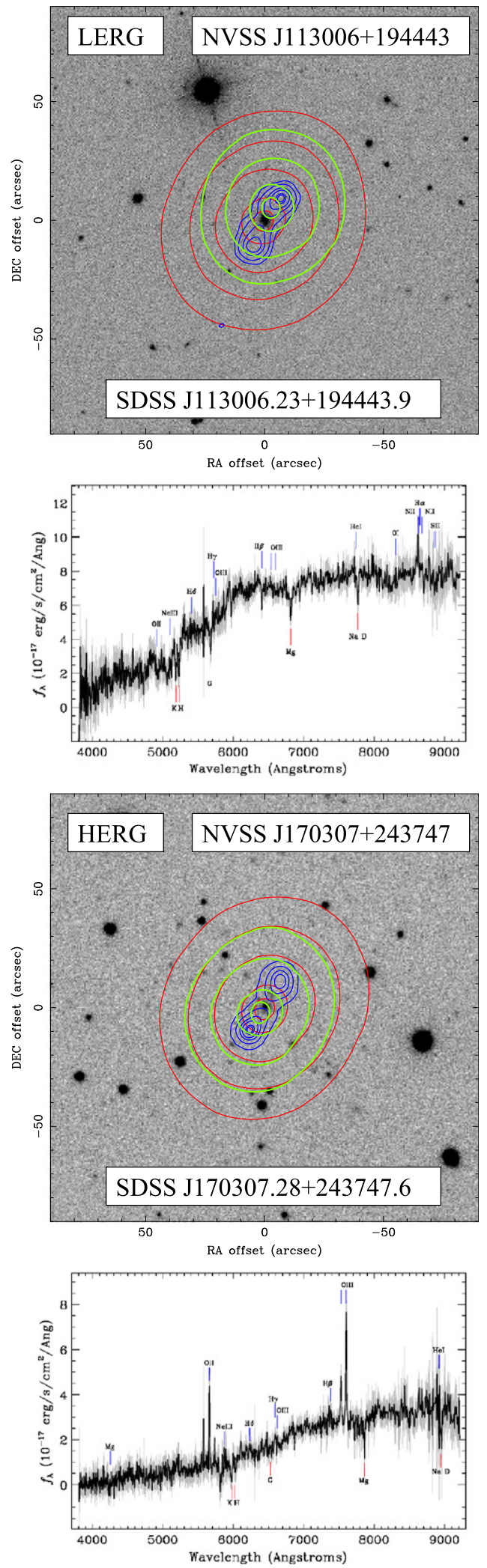

Figure 1. Example images from the cross-matched data sets, with the corresponding SDSS spectrum of the host galaxy included (obtained from the SDSS Science Archive Server, http://dr10.sdss3.org/). Top: low-excitation radio galaxy $($ LERG) at $($ R.A., decl. $)=(172.52602,19.74556)$. Bottom: highexcitation radio galaxy (HERG) at (R.A., decl.) $=(255.78027,24.62987)$. SDSS i-band (grayscale), NVSS total intensity (red contours), NVSS polarized intensity (green contours), FIRST total intensity (blue contours). 
sources into FR1 and FR2 based on whether the source was edge-brightened with distinct hotspots (FR2) or edge-darkened with the highest surface brightness features along the jet and/or the core (FR1). To ensure that our results are not influenced by misclassifications, we denote source morphologies as "FR1?" or "FR2?" in the cases where the FIRST image fidelity is not of sufficient quality to conclusively decide whether it really is an FR1 or FR2. We assign an FR0 classification (e.g., Sadler et al. 2014; Baldi et al. 2015) to those sources that are unresolved at $5^{\prime \prime}$. This corresponds to a projected linear extent of $\sim 15 \mathrm{kpc}$ at the median redshift of our sample $\left(z_{\text {median }}=0.2\right)$. Thus, these sources are most likely compact symmetric objects, blazars or sources with weak jets (e.g., low-luminosity AGN). The sources classified as 'FR0?' are ones that have a bright core but for which it is unclear if there is extended emission that has been resolved out or is too faint to be detected in FIRST. In addition to the FR0/FR1/FR2 classification, we also manually classified the radio morphology into "straight," "bent," and "compact." Sources were determined to be "straight" or "bent" depending on whether the extended emission on either side of the host galaxy was co-linear or not. The criterion for the "compact" classification is identical to the FR0 classification. To calculate the projected linear size of the source in kiloparsecs, we use a manual estimate of the angular size of each source along with the redshift from the SDSS. When the angular size of a source was larger than the synthesized beam of the FIRST image, we estimated the full angular extent of the emission by drawing a straight line between the extremities of the emission. Unresolved sources were assigned an angular size of $5^{\prime \prime}$, which corresponds to an upper limit on the linear size of the source.

\subsection{Infrared (IR) Data}

We also cross-matched our sample of polarized HERGs and LERGs with the Wide-field Infrared Survey Explorer (WISE; Wright et al. 2010), conducted at four bands, 3.4, 4.6, 12, and 22 $\mu \mathrm{m}$, with $5 \sigma$ point source sensitivities in unconfused regions of $\sim 0.08,0.11,1.0$, and $6.0 \mathrm{mJy}$, and angular resolutions of 6.1 , $6.4,6.5$, and 12 arcsec, in each band respectively. The IR properties across the WISE band are useful in determining the host galaxy types of our sample (e.g., quiescent, star-forming). We found 456 reliable cross-matches out of 796 sources down to a $5 \sigma$ detection level in at least one of the four WISE bands. For conversion from the calibrated WISE VEGA magnitudes to flux density (in Jy), we followed the instructions on the WISE webpage, ${ }^{9}$ including the $10 \%$ flux correction in the $22 \mu \mathrm{m}$ band.

\section{RESULTS}

The integrated degree of polarization measured at $1.4 \mathrm{GHz}$ $\left(\Pi_{1.4 \mathrm{GHz}}\right)$ in the NVSS can be influenced by a number of effects. First, bright and resolved structures in radio galaxies (i.e., knots, filaments, hotspots) typically do not achieve degrees of polarization larger than $\sim 30 \%$ to $50 \%$ (Saikia \& Salter 1988). This implies at least some amount of intrinsic disorder to the magnetic field on small scales in order to reduce it from the theoretical maximum of $\sim 72 \%$ expected for optically thin synchrotron radiation in a completely uniform magnetic field (e.g., Pacholczyk 1970). ${ }^{10}$ Second, even with

\footnotetext{
9 http://wise2.ipac.caltech.edu/docs/release/allsky/expsup/

10 Note that a completely disordered field that is compressed can also produce a high degree of polarization (e.g., Laing 1981).
}

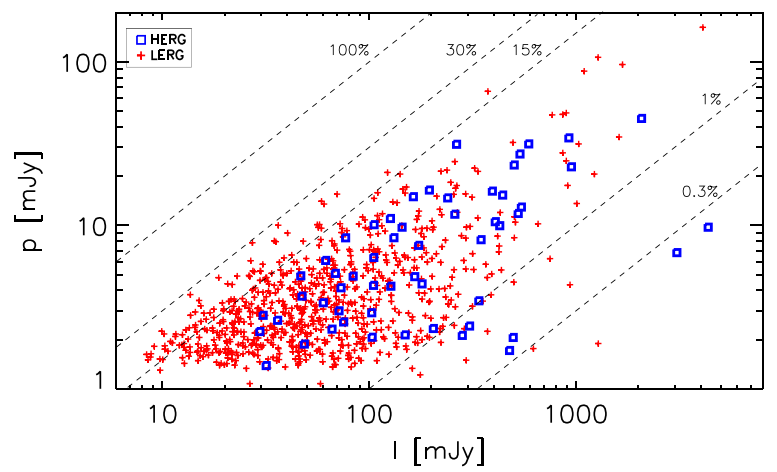

Figure 2. Integrated linearly polarized flux density $(p)$ vs. total flux density $(I)$ for HERGs (blue squares) and LERGs (red plus symbols). Diagonal dashed lines represent constant values of integrated degrees of polarization.

high intrinsic degrees of polarization on small scales, if the magnetic field structure is not globally uniform then polarization angle cancellation across the jet and lobe structure will significantly reduce the integrated degree of polarization. Third, polarization measurements at $1.4 \mathrm{GHz}$ are expected to be strongly affected by Faraday rotation, since the rotation of the polarization angle depends on wavelength squared. Large spatial fluctuations in Faraday rotation are observed in radio galaxies (e.g., Laing et al. 2008), which cause significant depolarization at $1.4 \mathrm{GHz}$. Finally, even if the intrinsic magnetic field is globally uniform and the Faraday rotation is negligible, observed asymmetries of the jet and lobe emission structure, due to physical bends or relativistic effects, will lead to polarization angle cancellation that can reduce the degree of polarization when integrated over the entire source.

For the 796 polarized sources, we find that $\Pi_{1.4 \mathrm{GHz}}$ ranges from $>0.1 \%$ to $\lesssim 30 \%$ (Figure 2 ), with a median value of $6.2 \%$. This is consistent with the maximum integrated polarization expected at $1.4 \mathrm{GHz}$ of $\lesssim 30 \%$ from surveys of extragalactic radio sources (e.g., Shi et al. 2010; Hales et al. 2014). Since our only available polarization measurements are at a single frequency and they have an angular resolution of $45^{\prime \prime}$ (corresponding to $\sim 150 \mathrm{kpc}$ at the median redshift of 0.2 of the sample), we cannot study the depolarization characteristics of our sample or the polarization morphology in any detail. Thus, we attempt to gain insight into the nature of HERG and LERG sources through their integrated degree of polarization properties at $1.4 \mathrm{GHz}$, in conjunction with their optical spectroscopic properties and total intensity radio morphology.

\subsection{Integrated Degree of Polarization of HERGs and LERGs}

Out of the 9863 LERGs and 481 HERGs identified by BH12 out to $z \sim 0.7$, we detect 741 LERGs and 55 HERGs with integrated linearly polarized intensity at $1.4 \mathrm{GHz}$ greater than $8 \sigma(\sim 1.6 \mathrm{mJy})$. There are 67 LERGs and 2 HERGs that have an integrated polarized flux density less than $1.6 \mathrm{mJy}$. These are still $8 \sigma$ detections but have lower local noise estimates than are typical for the entire NVSS. The faintest total intensity source we detect in polarization has a Stokes $I$ flux density of $8.6 \mathrm{mJy}$. At this flux level, there are 393 HERGs and 7661 LERGs, meaning that $\sim 90 \%$ of sources down to this flux level remain undetected in polarization. The integrated total and polarized flux densities of the detected polarized sources are shown in Figure 2 with HERGs identified as blue square symbols and LERGs as red plus symbols; the dashed lines are 


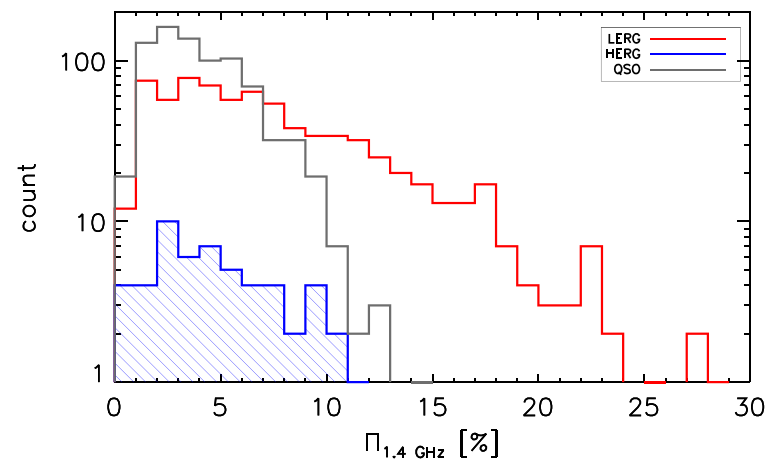

Figure 3. Histograms of the integrated degree of polarization $\left(\Pi_{1.4 \mathrm{GHz}}\right)$ for LERGs (red), HERGs (blue, hatched), and radio-loud QSOs (gray).

used to highlight the fact that the majority of polarized sources have integrated $1.4 \mathrm{GHz}$ percentage polarizations $\left(\Pi_{1.4 \mathrm{GHz}}\right)$ between $1 \%$ and $30 \%$. Both types of sources cover similar ranges in polarized intensity and the high fractional polarization sources are not all clustered at the detection limit (Figure 2). The rms residual instrumental polarization of the NVSS is estimated as $\sim 0.3 \%$ (Condon et al. 1998; Stil et al. 2014). Two LERGs and two HERGs have $\Pi_{1.4 \mathrm{GHz}}<0.3 \%$ (Figure 2), and we exclude them from our subsequent analysis. Thus, the remaining sample is composed of 53 HERGs and 739 LERGs.

The LERG sources span the full range of expected $\Pi_{1.4 \mathrm{GHz}}$ from $>0.3 \%$ to $\sim 30 \%$, while the detected HERGs are restricted to $\Pi_{1.4 \mathrm{GHz}}<15 \%$ (Figure 3 ). The median $\Pi_{1.4 \mathrm{GHz}}$ is $6.2 \%$ for LERGs and $4.2 \%$ for HERGs. A two-sided KolmogorovSmirnov (KS) test of $\Pi_{1.4 \mathrm{GHz}}$ for HERGs and LERGs gives a probability or " $p$-value" of $0.4 \%$, indicating a significant rejection, at approximately $2.9 \sigma$, of the null hypothesis (i.e., the two data sets are not likely drawn from the same underlying distribution). ${ }^{11}$ In the local universe, LERGs dominate over HERGs at low radio luminosity (BH12), meaning that LERGs are more numerous than HERGs at low radio flux density in our volume-limited sample (Figure 2). Ricean bias affects the measurement of polarized intensity more strongly at low flux density (see Section 2). Therefore, one might expect the LERGs to be more strongly affected by the polarization bias than the HERGs. If we restrict our sample to those sources with polarized intensity greater than $8 \sigma_{Q U}$, then we effectively eliminate the effect of the polarization bias (e.g., Simmons \& Stewart 1985). We also remove the 32 extended sources since the bias correction applied to them is not ideal for resolved sources. This reduces the sample to 43 HERGs and 474 LERGs, with a KS test of $\Pi_{1.4 \mathrm{GHz}}$ for these HERGs and LERGs now giving a $p$-value of $0.2 \%(\sim 3.1 \sigma)$. This small increase in significance shows that the polarization bias does not strongly affect our full sample.

A more important bias is the selection bias introduced by the threshold in polarized intensity at $\sim 1.6 \mathrm{mJy}$ (see Figure 2). This causes the median $\Pi_{1.4 \mathrm{GHz}}$ to increase for fainter sources because only sources with high values of $\Pi_{1.4 \mathrm{GHz}}$ will be detected. If we consider the sources for which this threshold in

\footnotetext{
11 Throughout this paper we use the two-sample KS test to determine the probability (or " $p$-value") that the null hypothesis can be rejected for two samples being tested. In all cases, the null hypothesis is that the two samples are drawn from the same underlying distribution. In addition to the $p$-value, we also quote the significance level in terms of the equivalent result from a normally distributed process.
}

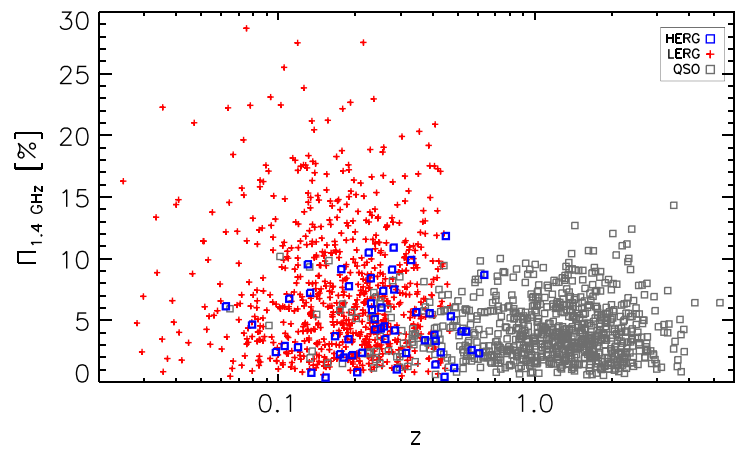

Figure 4. Integrated degree of polarization at $1.4 \mathrm{GHz}\left(\Pi_{1.4 \mathrm{GHz}}\right)$ vs. redshift (z) for LERGs (red), HERGs (blue, hatched), and radio-loud QSOs (gray).

polarized intensity is not as important (e.g., for sources brighter than $100 \mathrm{mJy}$ ), then we find no statistically significant difference between $\Pi_{1.4 \mathrm{GHz}}$ for HERGs and LERGs (see the Appendix). More sensitive polarization observations are required (by at least an order of magnitude) to conclusively test for a significant difference in $\Pi_{1.4 \mathrm{GHz}}$ between HERGs and LERGs down to $I \sim 10 \mathrm{mJy}$. However, Stil et al. (2014) circumvented this problem somewhat by stacking NVSS sources in polarized intensity to obtain the median degree of polarization for sources that were too faint to be detected. They found that the median degree of polarization increases with decreasing flux density $\left(\Pi_{1.4 \mathrm{GHz}} \propto S_{1.4 \mathrm{GHz}}^{-0.051}\right.$, where $S_{1.4 \mathrm{GHz}}$ is the NVSS flux density at $1.4 \mathrm{GHz}$ ). Our results suggest that one of the most likely causes of this increase in $\Pi_{1.4 \mathrm{GHz}}$ is the large number of LERGs at low flux with $\Pi_{1.4 \mathrm{GHz}}>15 \%$. Thus, the main focus of this paper is on the absence of HERGs with $\Pi_{1.4 \mathrm{GHz}}>15 \%$ and the ability of some LERGs to achieve values of $\Pi_{1.4 \mathrm{GHz}}$ up to $\sim 30 \%$.

\subsubsection{Integrated Degree of Polarization of Radio-loud QSOs}

Hammond et al. (2012) presented a catalog of 4003 polarized radio sources with redshifts, 815 of which were identified as QSOs in the SDSS, with 89 at $z<0.5$. Radio-loud QSOs are the equivalent of HERGs (i.e., radio-loud AGN with a radiatively efficient accretion disk) but with a more direct line of sight to the AGN and typically more luminous in the optical, allowing them to be more easily detected out to high redshift (e.g., van Velzen et al. 2015). A clear difference between the 1.4 $\mathrm{GHz}$ fractional polarization distributions of SDSS galaxies and of QSOs was found by Hammond et al. (2012), with the SDSS QSOs not exceeding fractional polarizations greater than $\sim 15 \%$ while the SDSS galaxies could reach as high as $\sim 30 \%$. Here we have essentially expanded on their sample of SDSS galaxies and separated them into the more physically meaningful classes of HERGs and LERGs. In Figure 3, separate histograms of $\Pi_{1.4 \mathrm{GHz}}$ are shown for the LERGs, HERGs, and QSOs. Now there are roughly equal numbers of radiative-mode AGN (i.e., HERGs and QSOs) and jet-mode AGN (i.e., LERGs), and the maximum degree of polarization of the radiative-mode $\mathrm{AGN}$ is still limited to $\lesssim 15 \%$.

This strongly suggests a fundamental difference between the polarization properties of radiative-mode AGN and jet-mode AGN at $1.4 \mathrm{GHz}$. We note that there are potentially strong redshift and evolutionary effects for the radio-loud QSO sample $(0.06<z<5.3)$. Thus, we limit most of our detailed analysis and discussion to the polarized HERGs and LERGs because they are similarly distributed in redshift, with 


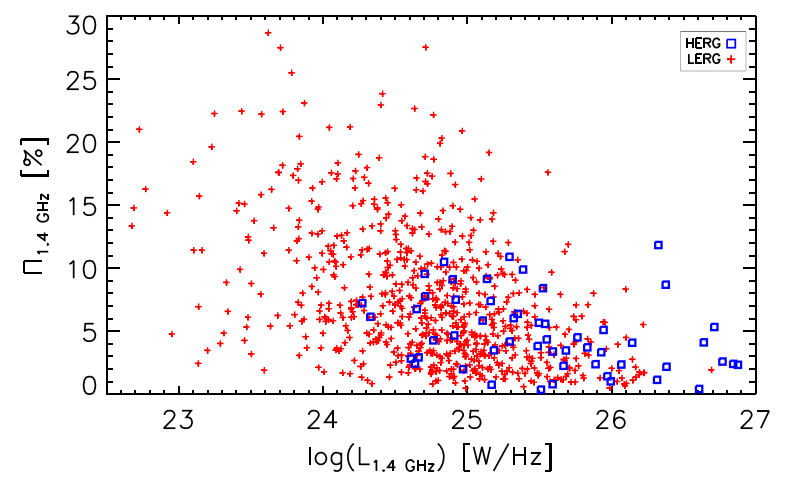

Figure 5. Integrated degree of polarization at $1.4 \mathrm{GHz}\left(\Pi_{1.4 \mathrm{GHz}}\right)$ vs. the $1.4 \mathrm{GHz}$ radio luminosity, in units of $\mathrm{W} \mathrm{Hz}^{-1}$, for HERGs (blue squares) and LERGs (red plus symbols). Note that we are insensitive to many lowluminosity, low- $\Pi_{1.4 \mathrm{GHz}}$ sources due to the polarization detection limit.

$z_{\text {median, HERG }}=0.25$ and $z_{\text {median, LERG }}=0.18$ (Figure 4$)$. In the Appendix, we replot Figure 4 for the sources with Stokes $I>100$ mJy only.

\subsection{Radio Luminosity Distribution of Polarized HERGs and LERGs}

The distribution of $\Pi_{1.4 \mathrm{GHz}}$ with $1.4 \mathrm{GHz}$ radio luminosity $\left(L_{1.4 \mathrm{GHz}}\right)$ is shown in Figure 5 . We calculate the radio luminosity using $L_{1.4 \mathrm{GHz}}=4 \pi D_{L}^{2} S_{1.4 \mathrm{GHz}}(1+z)^{-(\alpha+1)}$, where $D_{L}$ is the luminosity distance, $S_{1.4 \mathrm{GHz}}$ is the integrated total intensity and we have assumed a spectral index $\alpha=-0.7 .^{12}$ In the local universe, LERGs dominate the radio-loud AGN number counts at low luminosities $\left(22 \lesssim \log \left(L_{1.4 \mathrm{GHz}}\left[\mathrm{W} \mathrm{Hz}{ }^{-1}\right]\right) \lesssim 25\right)$, while above $L_{1.4 \mathrm{GHz}} \sim$ $10^{26} \mathrm{~W} \mathrm{~Hz}^{-1}$, HERGs begin to dominate (BH12). While examples of both populations are found across the full range of HERG and LERG luminosities, we only detect polarized HERGs above $L_{1.4 \mathrm{GHz}} \sim 10^{24} \mathrm{~W} \mathrm{~Hz}^{-1}$.

Since LERGs are more numerous than HERGs at lower luminosity and we have just shown that LERGs can achieve higher values of $\Pi_{1.4 \mathrm{GHz}}$, then one might expect an anticorrelation between $\Pi_{1.4 \mathrm{GHz}}$ and $L_{1.4 \mathrm{GHz}}$. We are insensitive to low-luminosity low- $\Pi_{1.4 \mathrm{GHz}}$ sources due to the polarization detection limit, and this strongly affects any robust inferences on any dependence of $\Pi_{1.4 \mathrm{GHz}}$ on $L_{1.4 \mathrm{GHz}}$. If we only consider sources with total intensities greater than $50 \mathrm{mJy}$, we have roughly $70 \%$ of both HERG and LERG sources below the detection limit in polarized flux density. This is roughly the limit at which useful statistics can be derived from data with upper limits (e.g., Antweiler \& Taylor 2008). Using a Kendall's tau rank correlation, we find a statistically significant ( $p$-value of $6 \times 10^{-8}, \sim 5.4 \sigma$ ), but weak, anticorrelation (correlation coefficient of -0.1 ) between $\Pi_{1.4 \mathrm{GHz}}$ and $L_{1.4 \mathrm{GHz}}$ for all sources. Considering the HERG sources only, we find no statistically significant correlation but we find a marginally significant anticorrelation between $\Pi_{1.4 \mathrm{GHz}}$ and $L_{1.4 \mathrm{GHz}}$ for the LERG sources ( $p$-value of $0.013 \%, \sim 3.8 \sigma$, for a correlation coefficient of -0.1). There is no evidence for an anticorrelation between $\Pi_{1.4 \mathrm{GHz}}$ and $L_{1.4 \mathrm{GHz}}$ for the brightest sources (i.e., with Stokes $I>100 \mathrm{mJy}$, as shown in the Appendix). This suggests that any anticorrelation between $\Pi_{1.4 \mathrm{GHz}}$ and $L_{1.4 \mathrm{GHz}}$

\footnotetext{
12 We define the spectral index, $\alpha$, such that the observed flux density $(S)$ at frequency $\nu$ follows the relation $S_{\nu} \propto \nu^{+\alpha}$.
}

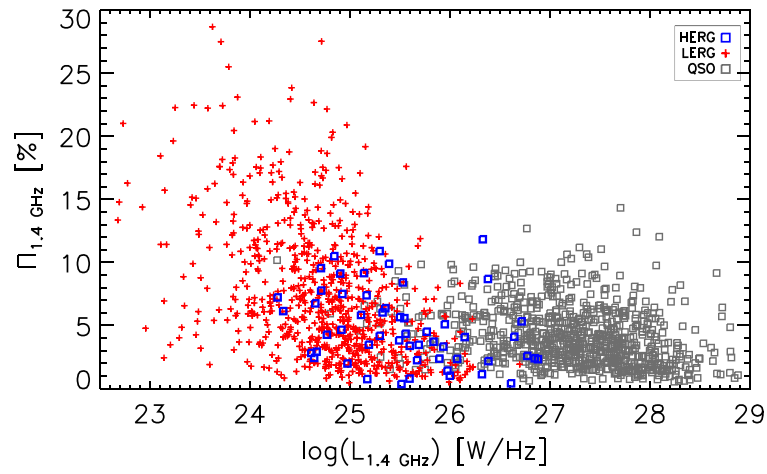

Figure 6. Integrated degree of polarization at $1.4 \mathrm{GHz}\left(\Pi_{1.4 \mathrm{GHz}}\right)$ vs. the 1.4 $\mathrm{GHz}$ radio luminosity, in units of $\mathrm{W} \mathrm{Hz}^{-1}$, for HERGs (blue squares), LERGs (red plus symbols), and radio-loud QSOs (gray squares).

is mainly driven by the change in the dominant population of radio-loud AGN toward lower luminosities from HERGs to LERGs. Much deeper radio polarization observations are required to more robustly test the existence of an anticorrelation between $\Pi_{1.4 \mathrm{GHz}}$ and $L_{1.4 \mathrm{GHz}}$ for the LERG sources. Of course, radio luminosity may be anticorrelated with $\Pi_{1.4 \mathrm{GHz}}$ independent of the HERG/LERG classification, and deeper polarization observations would also provide a key test for the continued absence of low-luminosity HERGs with $\Pi_{1.4 \mathrm{GHz}}>15 \%$.

It is interesting to extend this plot to higher radio luminosities by including all the known radio-polarized QSOs (Hammond et al. 2012) that overlap with the sky coverage of our sample (i.e., the SDSS area). Figure 6 shows $\Pi_{1.4 \mathrm{GHz}}$ versus $L_{1.4 \mathrm{GHz}}$ again but with the QSOs included (gray squares). This demonstrates the extension of the radiativemode AGN population to higher luminosities and clearly shows the limitation of radiative-mode AGN to values of $\Pi_{1.4 \mathrm{GHz}}<15 \%$, across five orders of magnitude in radio luminosity.

\subsection{Radio Morphology and Integrated Degree of Polarization}

We now present an investigation of the origin of the difference in $\Pi_{1.4 \mathrm{GHz}}$ for HERGs and LERGs in relation to the morphological properties of the large-scale radio emission (total intensity only, from the FIRST survey).

\subsubsection{FRO, FR1, and FR2}

Using our manual classification of FR0/FR1/FR2, described in Section 2.3, we find 14/6/18 HERGs that are FR0/FR1/FR2 with 15 uncertain classifications and 89/221/174 LERGs that are FR0/FR1/FR2 with 255 having an uncertain classification. It is immediately clear from this that the HERG/LERG class is essentially independent of the FR type. This is consistent with other studies by Best (2009), Lin et al. (2010), and Gendre et al. (2013). Figure 7 again plots $\Pi_{1.4 \mathrm{GHz}}$ versus radio luminosity but now with the FR type identified. It is clear from this plot that the range of $\Pi_{1.4 \mathrm{GHz}}$ is not restricted to values less than $15 \%$ for FR1, FR2, or FR0 sources. Thus, the HERGLERG difference in $\Pi_{1.4 \mathrm{GHz}}$ cannot be easily explained by the type of radio morphology. Similar results are obtained if we only consider the brightest sources, with $I>100 \mathrm{mJy}$ (see the Appendix). Figure 8 shows the empirical cumulative distribution functions (ECDFs) for $\Pi_{1.4 \mathrm{GHz}}$ of FR0, FR1, and FR2 sources. Considering only the definitive FR1- and FR2-type 


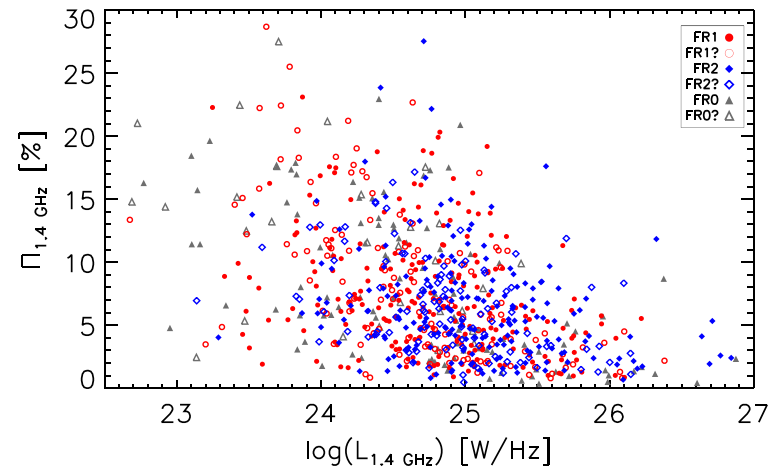

Figure 7. Integrated degree of polarization at $1.4 \mathrm{GHz}\left(\Pi_{1.4 \mathrm{GHz}}\right)$ vs. the $1.4 \mathrm{GHz}$ radio luminosity, in units of $\mathrm{W} \mathrm{Hz}^{-1}$, for FR0 (gray triangles), FR1 (red circles), and FR2 (blue diamonds) radio morphologies. Filled symbols represent robust classifications, with open symbols representing uncertain classifications. See Sections 2.3 and 3.3.1 for details.

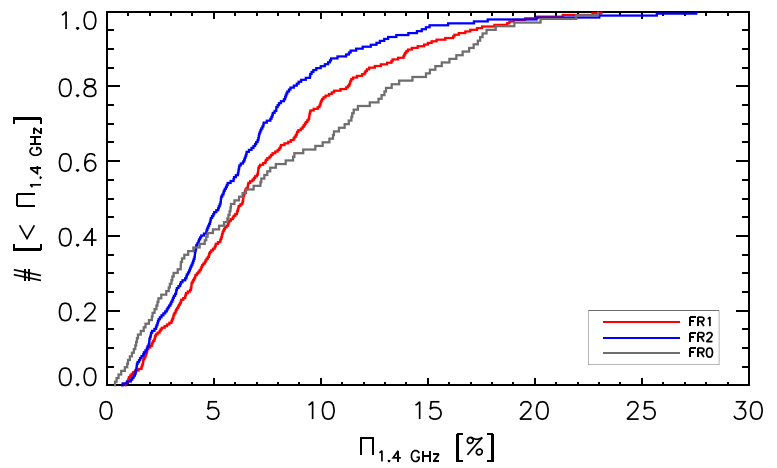

Figure 8. Empirical cumulative distribution functions (ECDFs) of the integrated degree of polarization $\left(\Pi_{1.4 \mathrm{GHz}}\right)$, for FR2 (blue), FR1 (red), and FR0 (gray) classifications.

sources, we find a marginally significant difference $(\sim 2.3 \sigma)$ in $\Pi_{1.4 \mathrm{GHz}}$ between the two types, with FR1s having a median $\Pi_{1.4 \mathrm{GHz}}$, FR1 of $6.4 \%$ and FR2s a median $\Pi_{1.4 \mathrm{GHz}}$, FR2 of $5.3 \%$. Similar results were previously found by Grant (2011) for a smaller number of sources (19 FR1s and 17 FR2s). We find no significant difference in $\Pi_{1.4 \mathrm{GHz}}$ between FR0 and FR1 sources ( $p$-value of $18 \%$ ) but get a $\sim 3 \sigma$ difference ( $p$-value of $0.3 \%$ ) in $\Pi_{1.4 \mathrm{GHz}}$ between FR0 and FR2 sources. The median $\Pi_{1.4 \mathrm{GHz}}$, FR0 of FR0 sources is $6.1 \%$.

\subsubsection{Straight Jets and Bent Jets}

Intrinsic polarization angle cancellation in radio sources with bent or disturbed morphologies could result in low integrated degrees of polarization, while the undisturbed, straight jet sources may achieve much higher values of $\Pi_{1.4 \mathrm{GHz}}$. Figure 9 presents ECDFs of $\Pi_{1.4 \mathrm{GHz}}$ split into straight, bent, and compact sources (as defined in Section 2.3). The majority of polarized sources are classified as straight (58\%), with $25 \%$ bent and the remaining $17 \%$ being compact. For sources with $\Pi_{1.4 \mathrm{GHz}}>15 \%$ the fraction of straight sources is $60 \%$ (similar to the fraction for all sources), but the fraction of bent sources falls to only $5 \%$. KS tests show that both compact and straight sources have significantly different $\Pi_{1.4 \mathrm{GHz}}$ distributions than bent sources ( $\sim 4.7 \sigma$ and $4.3 \sigma$, respectively) while compact and straight sources do not differ significantly from each other ( $p$-value of $3 \%$ ). We find that $40 \%$ of HERGs are straight, $32 \%$ are bent, and $28 \%$ are compact, while $60 \%$ of LERGs are straight with $24 \%$ bent, and $16 \%$ compact.

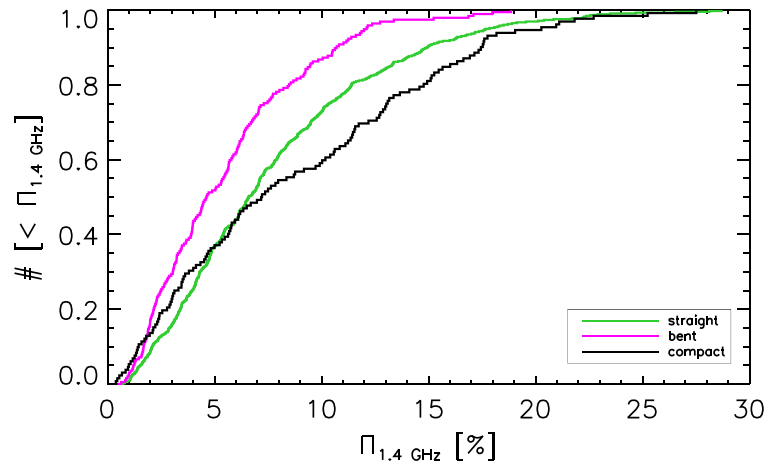

Figure 9. Empirical cumulative distribution functions (ECDFs) of the integrated degree of polarization at $1.4 \mathrm{GHz}\left(\Pi_{1.4 \mathrm{GHz}}\right)$ for radio morphologies classified as straight (green), bent (magenta), and compact (black). See Sections 2.3 and 3.3.2 for details.

\subsubsection{Influence of the "Radio Core"}

The contribution of radio emission from active or "restarted" emission regions near the central engine (i.e., the radio core) could decrease the integrated degree of polarization. This is possible because the inner regions of the radio source should suffer larger amounts of depolarization due to being more deeply embedded in the host galaxy. Furthermore, synchrotron self-absorbed and optically thick emission regions can only reach intrinsic degrees of polarization of approximately $10 \%$ (e.g., Pacholczyk 1970). In this case the additional total flux density provided by the core is likely to contribute very little additional polarized flux, causing the overall degree of polarization to decrease. To investigate whether or not this was an important effect for our sample, we determined the number of extended sources (i.e., with angular sizes $>10^{\prime \prime}$ ) that also had radio cores in the FIRST images. Out of the 38 polarized HERGs with angular sizes greater than $10^{\prime \prime}$, six of them have radio cores $(\sim 16 \%)$. The median value of $\Pi_{1.4 \mathrm{GHz}}$ for these six sources is $3.7 \%$, while the median value of $\Pi_{1.4 \mathrm{GHz}}$ for the other 32 extended sources without a core is $4.4 \%$. This indicates that the presence of a core may have a small effect in reducing $\Pi_{1.4 \mathrm{GHz}}$ for HERGs (although a twosided KS test finds no significant difference in $\Pi_{1.4 \mathrm{GHz}}$ for HERGs with and without a core, $p$-value of 58\%). Of the 597 extended LERGs, 137 have radio cores $(\sim 23 \%)$. This shows that the presence of a core is slightly more likely for LERGs than HERGs. The median value of $\Pi_{1.4 \mathrm{GHz}}$ for LERGs with cores is $6.6 \%$ and that without cores is slightly lower, at $6.1 \%$. In fact, there are 17 LERGs with radio cores that have $\Pi_{1.4 \mathrm{GHz}}>15 \%$, showing that the presence of a core is not a hindrance to high integrated degrees of polarization; these cores may be dominated by bright, highly polarized, optically thin inner jet regions.

\subsubsection{Projected Linear Size}

One might expect the extended polarized emission of sources with larger linear sizes to have less Faraday depolarization, and thus higher $\Pi_{1.4 \mathrm{GHz}}$, due to the propagation of the radiation through a presumably less dense magnetoionic environment (e.g., Hardcastle \& Krause 2014). To investigate this possibility, we compare the projected linear extent of the radio emission in the source rest frame, measured as described in Section 2.3, to $\Pi_{1.4 \mathrm{GHz}}$, for the HERG and LERG sources (Figure 10). For all sources, there is no obvious strong 


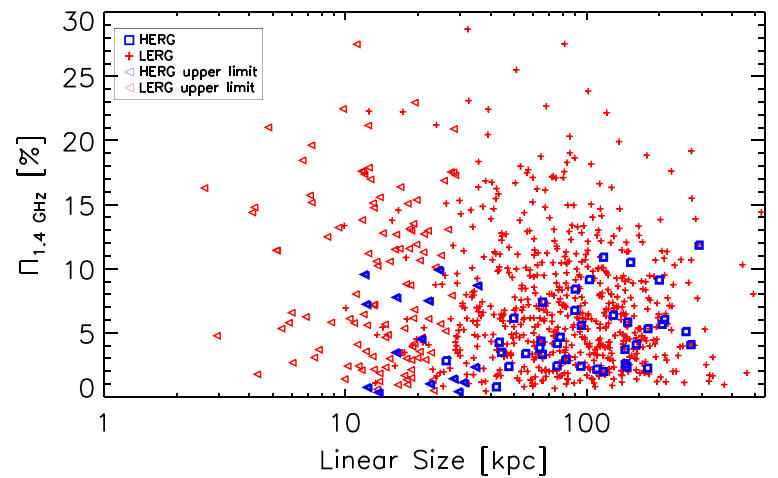

Figure 10. Integrated degree of polarization at $1.4 \mathrm{GHz}\left(\Pi_{1.4 \mathrm{GHz}}\right)$ vs. projected linear source size in $\mathrm{kpc}$, for LERGs (red plus symbols, with upper limits denoted by red triangles) and HERGs (blue squares, with upper limits denoted by blue triangles). See Sections 2.3 and 3.3.3 for details.

dependence of $\Pi_{1.4 \mathrm{GHz}}$ on linear size. Excluding the sources with upper limits, the median linear size of the HERG sample is $102.8 \mathrm{kpc}$, and that of the LERG sample is $82.8 \mathrm{kpc}$. Thus, if the Hardcastle \& Krause (2014) simulation results were true for all sources, then one would expect $\Pi_{1.4 \mathrm{GHz}}$ for HERGs to extend to higher values than the LERGs, completely the opposite to what is observed. A two-sided KS test indicates, however, that the linear size distribution of the HERGs is not significantly different from that of the LERGs ( $p$-value of $17 \%)$. The median linear size of sources with $\Pi_{1.4 \mathrm{GHz}}>15 \%$ is $64.7 \mathrm{kpc}$, with a $\mathrm{KS}$ test indicating a significant difference in linear size compared to sources with $\Pi_{1.4 \mathrm{GHz}}<15 \%$ ( $p$-value of $0.6 \%, \sim 2.7 \sigma)$. This surprisingly indicates that the most highly polarized LERGs are the ones with smaller projected linear extents (Figure 11). If we include the upper limits as measurements then the median linear size decreases to $75.4 \mathrm{kpc}$ for HERGs and $72.0 \mathrm{kpc}$ for LERGs. The larger decrease for HERGs occurs because a greater fraction of HERG sources are compact (cf. Section 3.3.2).

We caution that the smaller linear size estimates in the fainter NVSS sources may be confounded by a smaller estimate of the angular size due to the extended emission being resolved out/ undetected in FIRST. Furthermore, while the measurement of $\Pi_{1.4 \mathrm{GHz}}$ is independent of the FIRST angular size measurement, it is worth considering here whether or not the NVSS angular size influences the measurement of $\Pi_{1.4 \mathrm{GHz}}$ differently for HERGs or LERGs, even though the majority of sources are unresolved (84\%). However, a KS test of NVSS angular size for HERGs and LERGs shows no significant difference ( $p$ value of $26 \%$ ).

\subsection{Environmental Diagnostics of HERGs and LERGs}

Differences in the large-scale gaseous and magnetoionic environments are prime candidates for explaining the difference in $\Pi_{1.4 \mathrm{GHz}}$ for HERGs and LERGs, since the emission and morphology of the radio jet are expected to be strongly influenced by its local environment (e.g., Laing \& Bridle 2014). Here we explore several potential tracers of the environment local to the source.

\subsubsection{Faraday Rotation Measure}

The integrated fractional polarization at $1.4 \mathrm{GHz}$ is expected to be strongly affected by Faraday rotation, where magnetoionic material along the line of sight causes a rotation of the

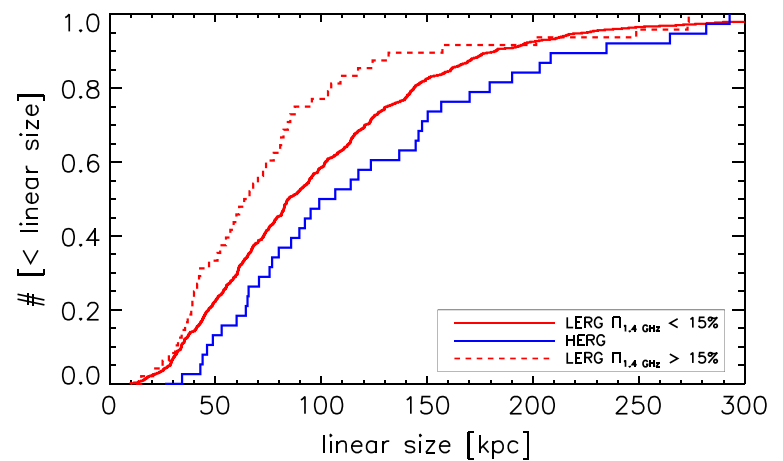

Figure 11. Empirical cumulative distribution function (ECDF) of the source linear size in kpc (excluding sources with upper limits), for LERGs with $\Pi_{1.4 \mathrm{GHz}}<15 \%$ (red solid line), HERGs (blue solid line), and LERGs with $\Pi_{1.4 \mathrm{GHz}}>15 \%$ (red dashed line).

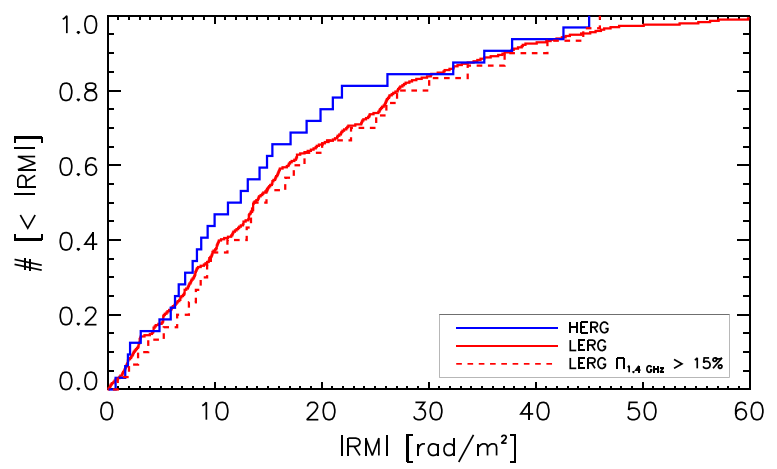

Figure 12. Empirical cumulative distribution function (ECDF) of the absolute value of the Faraday rotation measure $(|\mathrm{RM}|)$ in $\mathrm{rad} \mathrm{m}^{-2}$, for LERGs (red solid line), HERGs (blue solid line), and LERGs with $\Pi_{1.4 \mathrm{GHz}}>15 \%$ (red dashed line).

intrinsic linear polarization angle $(\Psi)$ with wavelength squared, $\Delta \Psi=\mathrm{RM} \lambda^{2}$. The Faraday rotation measure (RM) is defined as $0.81 \int_{L}^{0} n_{e} B_{\|} d l \mathrm{rad} \mathrm{m}^{-2}$, where $n_{e}$ is the electron number density in $\mathrm{cm}^{-3}, B_{\|}$is the line-of-sight magnetic field strength in $\mu \mathrm{G}$ and $L$ is the distance through the magnetoionic region in parsecs. For example, with $B_{\|} \sim 1 \mu \mathrm{G}, n_{e} \sim 10^{-4} \mathrm{~cm}^{-3}$ and $L \sim 100 \mathrm{kpc}$ this gives an $\mathrm{RM}$ of $\sim \pm 8 \mathrm{rad} \mathrm{m}^{-2}$, which causes a polarization angle rotation of $20^{\circ}$ at $1.4 \mathrm{GHz}$. Fluctuations in RM of this order (and larger) across the jets and lobes of radio galaxies are not uncommon (e.g., Laing et al. 2008; Guidetti et al. 2012). Thus, we expect such RM fluctuations local to the radio source to strongly reduce the integrated fractional polarization at $1.4 \mathrm{GHz}$.

In order to investigate the relative importance of this for HERGs and LERGs, we use the RM catalog of Taylor et al. (2009), which produced $37,543 \mathrm{RMs}$ by reprocessing the NVSS data into two channels centered on 1.365 and $1.435 \mathrm{GHz}$. Using this catalog, we can compare the RM of the integrated polarized emission for our sample of HERGs and LERGs. In Figure 12, we plot the ECDFs of the absolute value of the RMs for 295 LERGs and 34 HERGs $^{13}$. A two-sided KS test finds no significant difference between the two distributions ( $p$-value of 95\%). Furthermore, we also find no significant difference between the $|\mathrm{RM}|$ of sources with

\footnotetext{
13 The reason we did not find RMs for all our sources is that the Taylor et al (2009) RM catalog uses a higher threshold of $8 \sigma_{Q U}(\sim 2.3 \mathrm{mJy})$.
} 
$\Pi_{1.4 \mathrm{GHz}}>15 \%$ and those with $\Pi_{1.4 \mathrm{GHz}}<15 \%$ ( $p$-value of $99 \%)$. We searched for differences in $|\mathrm{RM}|$ between FR types, between straight and bent sources and in relation to linear size, finding no significant difference or dependence on any of the sub-samples. This is somewhat unsurprising since we know that the RMs from Taylor et al. (2009) are dominated by the Galactic contribution (Schnitzeler 2010; Stil et al. 2011). Attempts to model the Galactic RM component and subtract it from the total RM to robustly recover the extragalactic contribution are not possible with the current limited data due to poor constraints on individual source errors (e.g., Oppermann et al. 2015). However, this is expected to be possible in future with higher-precision RM measurements from observations with much broader and more continuous wavelengthsquared coverage.

\subsubsection{Depolarization Due to the Local Environment}

As described above, fluctuations in the magnetoionic medium across the source can significantly depolarize the radio emission. The recent study of Farnes et al. (2014) presented a parameterization of the change in the degree of polarization with wavelength (i.e., a degree of polarization spectral index, $\beta$, where $\Pi_{\lambda} \propto \lambda^{\beta}$ ) for 951 radio sources. They found that sources with a steep total intensity spectrum exhibited depolarization (a decreasing degree of polarization with increasing wavelength), while flat-spectrum sources typically maintain approximately constant degrees of polarization over large ranges in wavelength. From this they concluded that $\beta$ is predominantly affected by the magnetoionic environment local to the source, rather than by material in the distant foreground (i.e., intergalactic magnetic fields and/or the Milky Way). Only twelve of the sources presented here have an estimate for $\beta$ from Farnes et al. (2014), with $\beta$ ranging from -0.8 to 0.3 , with a median value of -0.2 . While this is insufficient for a robust statistical analysis of our sample, we can use the overall results of Farnes et al. (2014) to infer that $\Pi_{1.4 \mathrm{GHz}}$ for the majority of our sources, which have steep total intensity spectral indices, are likely affected by depolarization due to the local environment. Further observations are required to determine if HERGs and LERGs have different depolarization properties.

\subsubsection{Galaxy Number Density}

We now consider the polarization properties of HERGs and LERGs in comparison with one of the most direct measures of environment, galaxy number density. Estimates of the galaxy number density are available for $\sim 20 \%$ of our polarized sources, from Lin et al. (2010). They estimated the excess number of galaxies over the mean background within $0.5 \mathrm{Mpc}$ $\left(\Sigma_{0.5 \mathrm{Mpc}}\right)$ of the host galaxies of 1040 extended FIRST sources. For the polarized HERGs and LERGs, the median value of $\Sigma_{0.5 \mathrm{Mpc}}$ is 6.6 , compared to the unpolarized sources, which have a median $\Sigma_{0.5 \mathrm{Mpc}}$ of 8.8 , with a $\mathrm{KS}$ test indicating a significant difference ( $p$-value of $4.8 \times 10^{-5}, \sim 4 \sigma$ ). This means that the polarized sources are typically in more underdense environments than the unpolarized sources. The median value of $\Sigma_{0.5 \mathrm{Mpc}}$ for all (polarized and unpolarized) HERGs is 4.1 compared to 8.6 for all LERGs, supporting previous studies that found that HERGs are generally in less dense environments than LERGs (e.g., Best 2004). A KS test of $\Sigma_{0.5 \mathrm{Mpc}}$ for polarized HERGs versus polarized LERGs finds

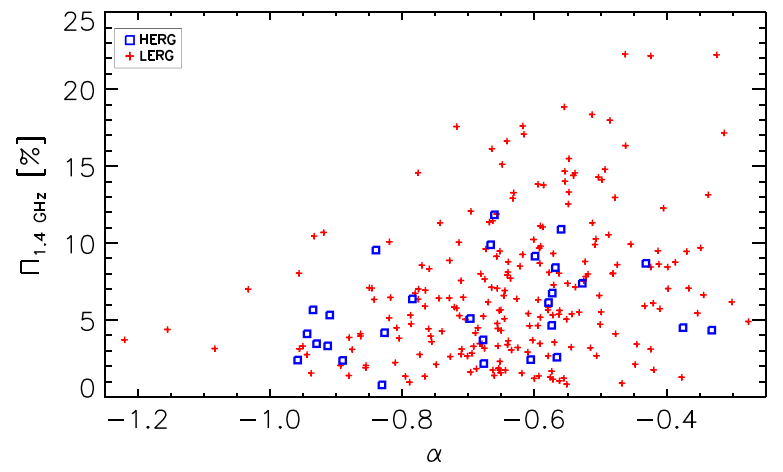

Figure 13. Integrated degree of polarization at $1.4 \mathrm{GHz}\left(\Pi_{1.4 \mathrm{GHz}}\right)$ vs. the total intensity spectral index $(\alpha)$ taken from Farnes et al. (2014), for HERGs (blue squares) and LERGs (red plus symbols). See Section 3.4.4 for details.

no significant difference between the sources ( $p$-value of 10\%). However, only five polarized HERGs have $\Sigma_{0.5}$ Mpc estimated, with all having values of $\Sigma_{0.5 \mathrm{Mpc}}<6.8$. For the LERGs, the median values of $\Pi_{1.4 \mathrm{GHz}}$ are $6.3 \%$ and $7.5 \%$ for sources with $\Sigma_{0.5 \mathrm{Mpc}}$ greater than and less than the median value of $\Sigma_{0.5 \mathrm{Mpc}}$, respectively ( $p$-value of $0.3 \%, \sim 2.9 \sigma$ significance). This suggests that, at least for the LERGs, the highest integrated degrees of polarization appear to favor environments with the lowest galaxy density.

\subsubsection{Spectral Index as a Probe of Environment Density}

Samples of radio galaxies selected by spectral index have previously shown that the steepest spectral index sources typically reside in rich clusters of galaxies (Baldwin \& Scott 1973; Slee et al. 1983). This has been interpreted as a result of pressure-confinement of the radio lobes propagating in dense environments (e.g., Klamer et al. 2006). We have total intensity spectral index measurements $(\alpha)$ from Farnes et al. (2014) for $32 \%$ of LERGs and $56 \%$ of HERGs, with $95 \%$ of LERGs and $100 \%$ of HERGs having $\alpha$ within the range $-1.5<\alpha<-0.3$. This supports the expectation that the majority of our polarized sources are indeed lobed radio galaxies with the dominant polarized emission presumably coming from the steep-spectrum, extended regions of the source (cf. Banfield et al. 2011; Hales et al. 2014).

From the plot of $\Pi_{1.4 \mathrm{GHz}}$ versus $\alpha$, shown in Figure 13, one can immediately notice the absence of sources with large $\Pi_{1.4 \mathrm{GHz}}$ and steep $\alpha$ (i.e., $\alpha<-0.7$ ). However, this appears to be true only for the LERG sources since the HERGs essentially uniformly cover their $\Pi_{1.4 \mathrm{GHz}}-\alpha$ parameter space. Using a Kendall's tau rank correlation test, we find a significant correlation between $\alpha$ and $\Pi_{1.4 \mathrm{GHz}}$ for the steep-spectrum LERGs, with a correlation coefficient of 0.2 at $\sim 4.5 \sigma$ significance. Employing the same correlation test for the HERGs only, we find no significant correlation. There are no known selection effects in Farnes et al. (2014) that could connect both the spectral index (derived from multiple surveys) and the polarization fraction (derived from just NVSS). Therefore, if very steep values of $\alpha$ are indicative of dense environments, then it is interesting to relate the absence of highly polarized LERG sources with $\alpha<-0.7$ to the effect of the environment. 


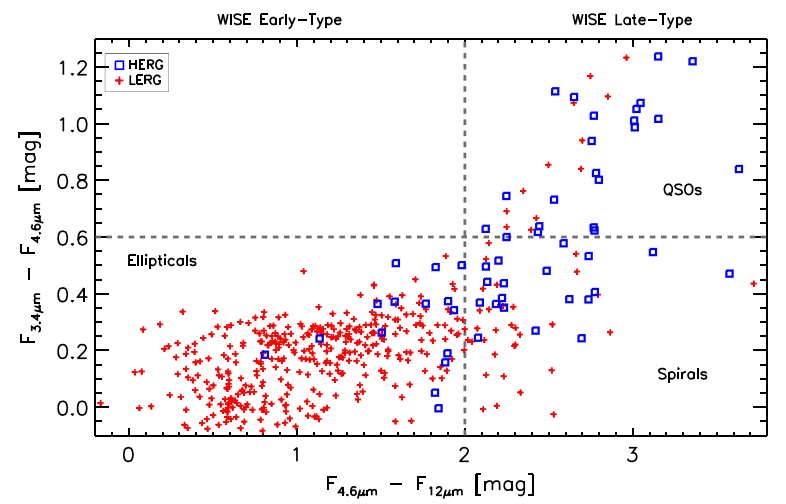

Figure 14. WISE infrared color-color plot for LERGs (red plus symbols) and HERGs (blue squares). The dashed lines denote the regions where different galaxy populations are expected to be. See Section 3.5.1 for more details.

\subsection{Host Galaxy and Accretion Rate Properties of Polarized HERGs and LERGs}

In order to infer general properties of the HERG and LERG populations from the subset of sources with significant integrated polarized emission at $1.4 \mathrm{GHz}$, we need to assess whether or not the polarized sources are generally representative of their individual classes. Previous studies have found that in the local universe HERGs are generally found in disk galaxies with ongoing star formation and with a high density of cold gas accreting at a rate greater than $\sim 1 \%$ of the Eddington rate. In contrast, LERGs are mainly found in massive elliptical galaxies with more massive black holes fueled by slowly cooling hot gas that accretes at less than $1 \%$ of the Eddington rate. The accretion energy of LERGs is mainly channeled into their jets, which in turn limit the amount of hot gas cooling in their host galaxy and cluster environments in a feedback cycle. See Heckman \& Best (2014) for a comprehensive review. In the remaining part of this section, we present the results of our analysis of the optical, infrared, and radio properties of the polarized HERG/LERG sample in order to determine their host galaxy and accretion rate properties.

\subsubsection{WISE Color-Color Host Galaxy Diagnostics}

The IR properties across the WISE band can be used to help separate early-type elliptical galaxies from galaxies with a substantial amount of ongoing star formation in disky/spiral galaxies as well as identifying those AGN with a bright accretion disk (i.e., a QSO). Figure 14 shows the WISE colorcolor plot for our sample. The dotted horizontal and vertical lines are from Wright et al. (2010), who divide elliptical and spiral galaxies at a WISE [4.6]-[12] color of $+1.5 \mathrm{mag}$ and find that the most powerful optical AGN lie above a [3.4]-[4.6] color of $+0.6 \mathrm{mag}$. From this we can see that the majority of LERGs are hosted by elliptical galaxies with only a small fraction classified as having spiral-type hosts. The LERGs classified as QSOs are potentially heavily obscured Type 2 AGN. The polarized HERGs are mainly in the WISE late-type category $(72 \%)$ with most of the rest $(22 \%)$ clustered within 0.5 mag of the dividing line between WISE early- and late-type categories. This is broadly consistent with the BH12 detailed study of the optical host galaxy properties, where HERG host galaxies were typically of lower stellar mass, lower black hole mass (i.e., less evolved) and had bluer colors than the LERGs.

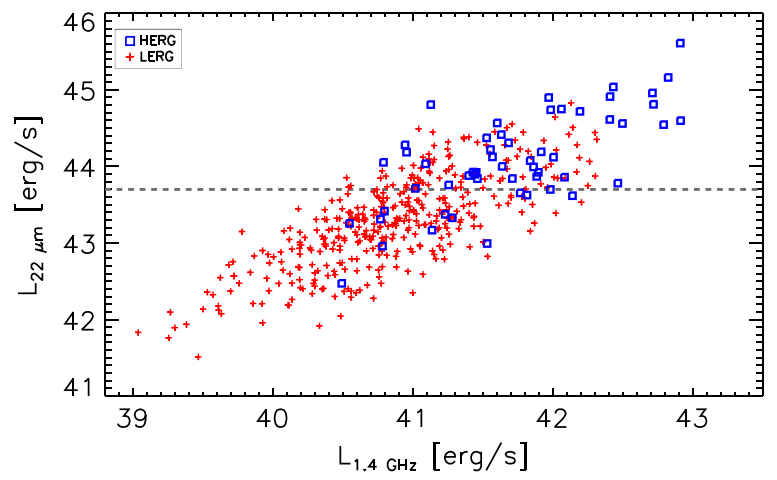

Figure 15. Mid-IR luminosity $\left(L_{22} \mu \mathrm{m}\right.$, in $\left.\mathrm{erg} \mathrm{s}^{-1}\right)$ vs. radio luminosity $\left(L_{1.4 \mathrm{GHz}}\right.$, in $\left.\mathrm{erg} \mathrm{s}^{-1}\right)$, for LERGs (red plus symbols) and HERGs (blue squares). The dashed line corresponds to the critical mid-IR luminosity of $5 \times 10^{43} \mathrm{erg} \mathrm{s}^{-1}$ defined by Gürkan et al. (2014). See Section 3.5.2 for details.

\subsubsection{WISE Mid-IR Luminosity}

Gürkan et al. (2014) analyzed the IR properties of a large sample (346) of radio-loud AGN including both nearby and high-redshift sources $(0.003<z<3.4)$. They found that their sample could be clearly divided into HERGs and LERGs in the mid-IR $(22 \mu \mathrm{m})$ radio-luminosity plane, with a critical mid-IR luminosity of $L_{22 \mu \mathrm{m}} \sim 5 \times 10^{43} \mathrm{erg} \mathrm{s}^{-1}$. If we divide our sample $(z<0.7)$ based on this mid-IR luminosity, we find that $75 \%$ of LERGs have $L_{22} \mu \mathrm{m}$ below $5 \times 10^{43} \mathrm{erg} \mathrm{s}^{-1}$ and $78 \%$ of HERGs have mid-IR luminosities above this value. Figure 15 shows there is a large scatter of 1 to 2 orders of magnitude about this dividing luminosity; however, it may be a potentially useful diagnostic tool in the absence of optical spectroscopy.

\subsubsection{Eddington-scaled Accretion Rate}

Recent studies have investigated the idea that a fundamental switch in the type of radio-loud AGN activity occurs at an accretion rate of $\sim 1 \%$ of the Eddington accretion rate (e.g., Mingo et al. 2014). At Eddington ratios greater than 1\%, the accretion disk structure is expected to represent the classical picture of an AGN in unified models with a geometrically thin accretion disk (e.g., Urry \& Padovani 1995), while at accretion rates much less than $1 \%$ of the Eddington rate there is a geometrically thick, radiatively inefficient accretion flow (e.g., Narayan \& Yi 1995).

In order to estimate the Eddington-scaled total accretion rate for the polarized HERGs and LERGs, we follow the approach adopted by BH12. They combined the optical bolometric luminosity, estimated from the $\mathrm{O}$ III emission line $\left(L_{\text {bol,O III }}\right)$, with the total mechanical luminosity of the radio jet $\left(L_{\text {mech }}\right)$, normalized to the Eddington luminosity $\left(L_{\text {Edd }}\right)$, to obtain the Eddington-scaled total accretion rate as $\left(L_{\text {mech }}+L_{\text {bol, OIII }}\right)$ / $L_{\text {Edd }}$.

To derive a mean bolometric correction for the SDSS [O III $]_{5007}$ emission line luminosity, Heckman et al. (2004) used a multi-wavelength analysis of a large sample of powerful Type 1 AGN to find

$$
L_{\mathrm{bol}, \mathrm{O} \text { III }} \approx 3500 L_{\mathrm{OIII}},
$$

with a scatter in $L_{\text {bol, }}$ III of \pm 0.38 dex. The $[\mathrm{O} \text { III }]_{5007}$ emission line is used because it is the strongest emission line in the majority of SDSS AGN, and has minimal contamination from star formation. Since this relation was derived mainly at low 
redshift $(z \sim 0.1)$ its applicability up to redshifts of $z \sim 0.5$ is questionable. However, Bernardi et al. (2003) found relatively little luminosity evolution in a study of 9000 early-type galaxies from the SDSS, in the redshift range $0<z<0.3$. Furthermore, the metallicity evolution in star-forming galaxies from $z \sim 0.1$ to $z \sim 0.4$ is $\sim 0.1$ dex (Lara-López et al. 2009). The metallicity evolution of early-type galaxies would not be expected to change more dramatically than this over our redshift range. Thus, considering the quoted uncertainty for $L_{\text {bol, OIII }}$ of \pm 0.38 dex, we do not expect Equation (1) to be strongly affected by changing abundances in our redshift range up to $z \sim 0.5$. However, we will also check the significance of our results after excluding sources with $z>0.2$.

To estimate the jet mechanical luminosity from the $1.4 \mathrm{GHz}$ radio luminosity, we use the relation of Cavagnolo et al. (2010),

$$
L_{\text {mech }}=7.3 \times 10^{36}\left(L_{1.4 \mathrm{GHz}} / 10^{24} \mathrm{~W} \mathrm{~Hz}^{-1}\right)^{0.7} .
$$

This scaling relation between the jet mechanical luminosity $\left(L_{\text {mech }}\right)$ and the $1.4 \mathrm{GHz}$ radio luminosity is derived from X-ray cavity measurements in mainly low-power radio galaxies with radio luminosities less than $10^{25} \mathrm{~W} \mathrm{~Hz}^{-1}$ (e.g., Bîrzan et al. 2008). The large scatter in the data $(\sim 0.8$ dex $)$ means that there is considerable uncertainty in the slope of this relation, which makes its application to high-power radio sources questionable, as well as potentially introducing a systematic error in $L_{\text {mech }}$ between the HERG and LERG samples because of their different luminosity distributions. However, Godfrey \& Shabala (2013) used an independent method for determining the total power of sources with radio luminosities greater than $10^{25} \mathrm{~W} \mathrm{~Hz}^{-1}$, specifically FR2-type sources. Despite the significantly different radiative efficiencies of FR2- and FR1-type radio sources, Godfrey \& Shabala (2013) surprisingly found very good agreement with the relation of Cavagnolo et al. (2010) up to $10^{28} \mathrm{~W} \mathrm{~Hz}^{-1}$. While this agreement is encouraging, the applicability of both the same normalization and slope of Equation (2) across such a wide range in radio luminosity remains uncertain, with further work on a larger number of sources across the full radio-jet luminosity range required.

In order to calculate the Eddington luminosity,

$$
L_{\text {Edd }}=1.3 \times 10^{31} M_{\mathrm{BH}} / M_{\odot} \mathrm{W},
$$

for each of our sources, we require an estimate of the black hole mass $\left(M_{\mathrm{BH}}\right)$. Using the velocity dispersion of the host galaxy $\left(\sigma_{*}\right)$ taken from the SDSS spectrum, $M_{\mathrm{BH}}$ can be estimated for a large fraction of our sample through the $M-\sigma_{*}$ relation (e.g., Tremaine et al. 2002). This relation is based on the well determined dependence of the stellar velocity dispersion of a galaxy bulge on its black hole mass, described by

$$
\log \left(M_{\mathrm{BH}} / M_{\odot}\right)=8.13+4.02 \log \left(\sigma_{*} / 200 \mathrm{~km} \mathrm{~s}^{-1}\right) .
$$

The SDSS stellar velocity dispersion estimates are obtained by fitting a spectral template across the rest-frame wavelength range $400-700 \mathrm{~nm}$, with emission line regions explicitly masked out. The velocity dispersion is only estimated for $z<0.4$ and we have excluded any values above $420 \mathrm{~km} \mathrm{~s}^{-1}$

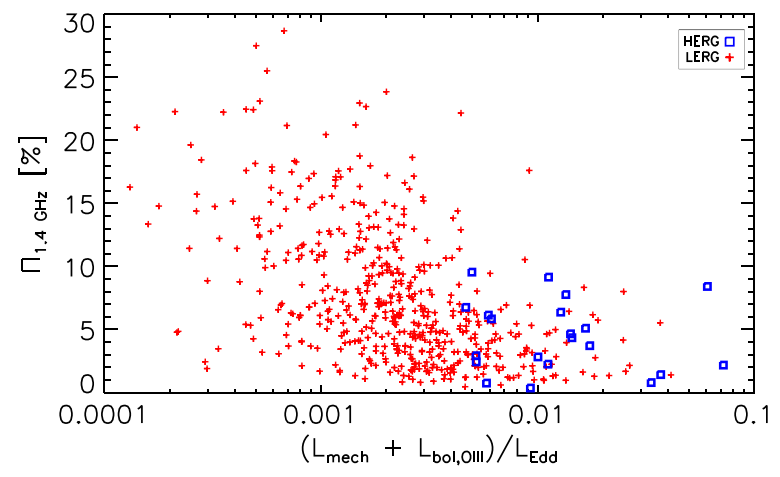

Figure 16. Integrated degree of polarization at $1.4 \mathrm{GHz}\left(\Pi_{1.4 \mathrm{GHz}}\right)$ vs. the total Eddington-scaled accretion rate, $\left(L_{\text {mech }}+L_{\text {bol,O III }}\right) / L_{\text {Edd }}$, for HERGs (blue squares) and LERGs (red plus symbols). See Section 3.5.3 for details.

and below $70 \mathrm{~km} \mathrm{~s}^{-1}$, since they cannot be reliably measured from the SDSS spectra. We also excluded any spectra with a median per-pixel signal-to-noise ratio of less than $10 .{ }^{14}$ This leads to 546 reliable velocity dispersion values (22 HERGs and 524 LERGs) with a median redshift of 0.15 and with only four sources with $z>0.3$. Thus, we find reliable velocity dispersion measurements for $40 \%$ of polarized HERGs and $71 \%$ of polarized LERGs. The lower fraction for HERGs is mainly due to a larger fraction of sources at higher redshift and with lower signal-to-noise ratio.

The stellar velocity dispersion as measured from the fixed $3^{\prime \prime}$ fiber aperture of the SDSS does not provide an accurate estimate of $M_{\mathrm{BH}}$ in disk-dominated galaxies because stars from the extended regions of the galaxy will be included. All the polarized HERGs and LERGs in our sample have a concentration index $(C=R 90 / R 50)$ greater than 2.6, where the parameter $R 90(R 50)$ represents the radius that encloses $90 \%(50 \%)$ of the host galaxy's optical light. A concentration index greater than 2.6 means that they are bulge-dominated systems (Shimasaku et al. 2001), as expected for the typical early-type hosts of radio-loud AGN. This means that the velocity dispersion as measured from the fixed $3^{\prime \prime}$ SDSS fibers $(\sim 8 \mathrm{kpc}$ at $z=0.15)$ is representative of the bulge in the majority of cases. However, at redshifts, $z>0.2$, the inclusion of the majority of the host galaxy's light may affect the velocity dispersion measurement and the $M_{\mathrm{BH}}$ estimate in a systematic manner. At redshifts, $z<0.1$ and $0.1<z<0.2$, we found median velocity dispersions of $240 \mathrm{~km} \mathrm{~s}^{-1}$ and $241 \mathrm{~km} \mathrm{~s}^{-1}$, respectively, while from $0.2<z<0.3$, the median velocity dispersion increases to $257 \mathrm{~km} \mathrm{~s}^{-1}$. This means that Eddingtonscaled accretion rates for sources with $z>0.2$ are typically $\sim 1.6$ times systematically larger than sources at $z<0.2$.

In Figure 16, we plot $\Pi_{1.4 \mathrm{GHz}}$ versus the Eddington-scaled total accretion rate, corresponding to $\left(L_{\text {mech }}+L_{\text {bol, OIII }}\right) / L_{\text {Edd }}$, for 22 polarized HERGs and 524 polarized LERGs. We see that the HERGs have systematically higher accretion rates $(\sim 0.5 \%-10 \%)$ than the majority of the LERGs, which have accretion rates $<1 \%$. In addition to this, it is clear that the more weakly accreting LERGs are more highly polarized than the HERGs and the strongly accreting LERGs. As noted by Mingo et al. (2014), the LERGs with Eddington ratios greater than

\footnotetext{
${ }^{14}$ See http://classic.sdss.org/dr2/algorithms/veldisp.html for details.
} 
$\sim 1 \%$ may be misidentified as radiatively inefficient sources and/or their radio luminosity is boosted by residing in dense environments. Thus, it is interesting to test the significance of the difference in $\Pi_{1.4 \mathrm{GHz}}$ between sources separated purely by their total Eddington ratios. A two-sided KS test indicates a highly significant difference in $\Pi_{1.4 \mathrm{GHz}}$ for sources separated at a total accretion rate of $0.5 \%$ of Eddington ( $p$-value of $1.9 \times 10^{-15}$, corresponding to $\sim 8 \sigma$ for a normally distributed process). We separated the sample at $0.5 \%$ of Eddington, instead of $1 \%$ of Eddington, in order to include all the HERG sources and better match the numbers in the two bins. If we exclude sources with $z>0.2$, due to their systematically higher Eddington luminosities, the KS test gives a $p$-value of $8.4 \times 10^{-9}(\sim 6 \sigma)$.

Mingo et al. (2014) found that the mid-IR bolometric luminosity $\left(L_{\mathrm{bol}, \mathrm{IR}}\right)$ provided a more reliable estimate of the accretion rate in HERGs. Thus, we use this as a consistency check against the accretion rates estimated using the $\left[\mathrm{O}_{\mathrm{III}}\right]$ emission line. From the WISE $22 \mu \mathrm{m}$ flux density, we can estimate $L_{\text {bol,IR }}$ from the scaling relation of Runnoe et al. (2012), where $\log \left(L_{\text {bol, IR }}\right)=15.035+0.688 \log \left(\lambda L_{\lambda}\right)$. While we find that $L_{\text {bol,IR }}$ can be up to an order of magnitude larger than $L_{\text {bol,O III }}$ for the HERGs, the qualitative difference when $L_{\text {bol,IR }}$ is used to estimate the total accretion rate instead of $L_{\text {bol,O III }}$ does not result in a significantly cleaner separation in the Eddington ratios between polarized HERGs and LERGs. This also does not hugely affect the significance of the difference in $\Pi_{1.4 \mathrm{GHz}}$ for sources separated at an Eddington ratio of $0.5 \%\left(p\right.$-value of $\left.1.3 \times 10^{-8}, \sim 5.7 \sigma\right)$.

The bolometric luminosity correction in Equation (1) is for the [O III] flux uncorrected for dust extinction. This correction can be important because while the $[\mathrm{O}$ III] line emission region lies outside the dusty torus of the AGN (i.e., in the narrow line region), it may still suffer significant amounts of extinction due to interstellar dust in the host galaxy. To investigate this potential error in $L_{\text {bol,O III }}$, we corrected for the effect of dust extinction on the $[\mathrm{O}$ III] flux using the Balmer decrement (i.e., the ratio of the fluxes of the $\mathrm{H} \alpha$ and $\mathrm{H} \beta$ narrow emission lines; see Lara-López et al. 2009 for details). The median size of the dust correction to the [O III] luminosity for our sample is $\sim 0.2$. Kauffmann \& Heckman (2009) used X-ray, optical, and midinfrared spectra of a complete flux-limited sample of SDSS type 2 AGN to derive a mean bolometric correction of 600 for the extinction-corrected [O III] luminosity. Using this bolometric correction to the extinction-corrected [O III] luminosity, instead of Equation (1), decreases the significance in $\Pi_{1.4 \mathrm{GHz}}$ between low and high Eddington ratios to $\sim 4.8 \sigma$. However, this decrease in significance is unsurprising since we lose a large fraction of our sources because we can only obtain the extinction correction for $\sim 50 \%$ of sources due to the absence of $\mathrm{H} \alpha$ and/or $\mathrm{H} \beta$ measurements.

While the different bolometric correction factors produce slightly different results and their application at redshifts $z>0.2$ is questionable, the main result of this section, that $\Pi_{1.4 \mathrm{GHz}}$ can achieve higher values for sources with lower total Eddington accretion rates, remains valid, for a separation at $\sim 0.5 \%$ of Eddington.

\section{DISCUSSION}

Our main observational result is the discovery of a difference in the integrated degree of polarization at $1.4 \mathrm{GHz}\left(\Pi_{1.4 \mathrm{GHz}}\right)$ between radio-loud radiative-mode $\mathrm{AGN}$ (i.e., HERGs and radio-loud QSOs) and jet-mode AGN (i.e., LERGs), with the weakly accreting sources extending to higher values of $\Pi_{1.4 \mathrm{GHz}}$. We now discuss the potential significance of these results in linking the large-scale host galaxy environment with the accretion state of the supermassive black hole and the production of powerful radio-loud jets.

\subsection{Environments of HERGs and LERGs}

At low redshift $(z<0.4)$, HERGs are found mainly in environments of low galaxy density (i.e., groups), while LERGs are found in a wide range of environments from poor to rich groups and clusters (e.g., Best 2004; Hardcastle 2004). They also differ in their host galaxy types, with HERGs mainly residing in galaxies with significant ongoing star formation implying an abundance of cold gas, and LERGs mainly hosted by massive elliptical galaxies with hot X-ray halos and a minimal supply of cold gas. This has led to the development of models claiming that the two different types of AGN result from their presence in different environments.

In particular, Hardcastle et al. (2007) proposed that the difference between HERGs and LERGs is due to the source of the accreting gas: Bondi accretion of the hot, X-ray gas in ellipticals for LERGs, and cold gas accretion from disk galaxies required for HERGs. Alternatively, the type of radio-loud AGN activity may instead be triggered by a fundamental change in the accretion disk structure at an Eddington-scaled accretion rate of $\sim 1 \%$ (e.g., Mingo et al. 2014), with a radiatively inefficient accretion flow below, and a radiatively efficent thin disk above, $\sim 1 \%$ of Eddington. However, these two scenarios are not mutually exclusive and both could potentially act together. It remains unclear as to what the exact nature of the relationship is between the large-scale environmental properties of AGN and the type of accretion onto their supermassive black holes.

In Section 3.5, we have shown that our sample of radiopolarized AGN display similar host galaxy properties and accretion rates as the full sample in BH12 and elsewhere (i.e., the host galaxies of our sample of HERGs typically have higher rates of star formation, lower black hole mass, and higher accretion rates than the LERGs). Thus, we can use our sample of polarized sources to reliably infer some of the key properties of the HERG and LERG populations in general.

The polarized sources in our sample are in less dense environments (in terms of the galaxy number density within $0.5 \mathrm{Mpc}$ ) than unpolarized sources. Furthermore, it is the most underdense environments of the polarized LERG sources that typically have higher values of $\Pi_{1.4 \mathrm{GHz}}$ (Section 3.4.3). With a similar approach, Shi et al. (2010) studied the most highly polarized sources in the NVSS and found a median galaxy count within a $1 \mathrm{Mpc}$ radius of $7 \pm 3$ (for only eight sources) compared to $9 \pm 7$ for a much larger low-polarization control sample. Their result is not inconsistent with our findings, and is suggestive of an influence of galaxy number density on $\Pi_{1.4 \mathrm{GHz}}$. Overall, estimates of galaxy number density seem to be crude but statistically useful probes of the environment densities of radio sources in relation to whether they are in a poor or rich group, or cluster.

In considering the radio source environment as a key variable in our analysis, we also need to recognize that radio sources with bent or disturbed morphology are often found in dense environments (e.g., Owen \& Rudnick 1976). In fact, radio sources with bent morphologies are used as a way to find 
candidate galaxy clusters (e.g., Wing \& Blanton 2011). In Section 3.3.2, we showed that bent radio sources have significantly lower values of $\Pi_{1.4 \mathrm{GHz}}$ than straight ones. We also found that $\Pi_{1.4 \mathrm{GHz}}$ for LERG sources was weakly correlated with the total intensity spectral index (Section 3.4.4), providing additional evidence that the LERGs with low values of $\Pi_{1.4 \mathrm{GHz}}$ are preferentially located in denser environments. This reduction in $\Pi_{1.4 \mathrm{GHz}}$ could be due to a combination of polarization angle cancellation, owing to asymmetric jet structure, as well as the large amounts of external Faraday rotation expected in dense environments, which would cause strong depolarization of the radio emission.

These results are generally consistent with Best (2004) who found, for $z<0.1$, a strong correlation between radio luminosity and environment richness for LERGs, but not for HERGs. Similarly, Ineson et al. (2013) found, for radio sources around $z \sim 0.4$, a weak correlation between radio luminosity and cluster richness for LERGs, but no correlation for HERGs. However, if the environment of radio sources does indeed strongly affect the integrated degree of polarization, the question still remains as to how exactly this is achieved. Thus, a more sensitive probe of the immediate environment of radio sources is desirable for probing the link between galaxy environment, black hole accretion, and powerful jet production in more detail.

One of the most sensitive probes of the local environment of radio sources is through Faraday rotation and its associated depolarization. In Sections 3.4.1 and 3.4.2, we showed that the currently available Faraday rotation and depolarization data for our sources are not sufficient to probe the local source environment in detail, but it is clear that the majority of depolarization occurs local to the source (e.g., Banfield et al. 2014; Farnes et al. 2014).

From our current investigation, we expect that the LERGs with high values of $\Pi_{1.4 \mathrm{GHz}}$ are suffering less depolarization in less dense environments than the LERGs with low values of $\Pi_{1.4 \mathrm{GHz}}$. This is supported by our observation that sources with straight jets are much more likely than bent jets to have $\Pi_{1.4 \mathrm{GHz}}>15 \%$ (Section 3.3.2), and the estimates of environment density discussed above indicate that the more highly polarized sources are more likely to exist in less dense environments. From simulations, the amount of depolarization is expected to decrease with increasing lobe size (e.g., Hardcastle \& Krause 2014). However, we find that the sources with $\Pi_{1.4 \mathrm{GHz}}>15 \%$ have smaller linear sizes (Section 3.3.3), possibly indicating that these LERGs also have intrinsically weaker jets. While the differences between FR1 and FR2 radio morphologies are generally attributed to a combination of jet power and how they interact with their ambient environments (i.e., the less powerful jets of FR1s are gradually decelerated by entrainment while the more powerful FR2s are not), our results in Section 3.3.1 suggest that the FR morphology is not the primary driver causing the observed difference in $\Pi_{1.4 \mathrm{GHz}}$ of the LERGs and HERGs.

Our claim that the density of the ambient environment is the key variable for the spread in $\Pi_{1.4 \mathrm{GHz}}$ for LERGs does not explain the fact that HERGs are limited to $\Pi_{1.4 \mathrm{GHz}}<15 \%$, since they are expected to exist in less dense environments, on average, than LERGs. A possible remedy to this could be related to the much greater radiative output of the HERG central engine. The high radiative output is likely photoionizing large amounts of the host galaxy interstellar medium and increasing the amount of "Faraday-active" material (i.e., the amount of magnetoionic material contributing to the total Faraday rotation as seen by the jet). The larger amount of magnetoionic material would also mean larger variations in Faraday rotation causing greater amounts of depolarization in HERGs, on average, than in LERGs. There is a well studied correlation between the AGN accretion disk luminosity and the amount of the host galaxy that is ionized (e.g., Netzer et al. 2006). In fact, in some cases there is evidence of saturation of the ionization radius, indicating that the AGN has potentially ionized the entire galaxy (e.g., Curran and Whiting 2010; Hainline et al. 2014). However, the central AGN need not be responsible for the ionization of the large-scale host galaxy environment in all cases. Extended emission line regions (EELRs), on scales of $\sim 100 \mathrm{kpc}$, are often found in sources with strong nuclear emission lines and are coincident with regions of high depolarization in the lobes of radio galaxies (e.g., Pedelty et al. 1989). There is also statistical evidence that these EELRs are aligned with the radio source axis and have similar extents to the radio lobes (McCarthy et al. 1987; Baum \& Heckman 1989). This suggests that the radio jet may also be responsible for the ionization of its immediate environment through shocks, and shock-induced star formation, as well as entrainment. Interestingly, from a Faraday RM study of 26 radio-loud AGN $(0.3<z<1.3)$, Goodlet \& Kaiser (2005) found that the difference in the RM of each lobe and the dispersion in RM both correlate with redshift, suggesting more dense magnetoionic environments at higher redshift. Since all their high-redshift sources are HERGs and their low-redshift sources are dominated by LERGs, this lends support to the argument that HERGs' maximum integrated polarization may be limited due to large amounts of Faraday depolarization local to the source. Once higherprecision Faraday RM and depolarization data are obtained for a large number of HERGs and LERGs, it would then be interesting to consider other factors, in addition to the magnetoionic environment, such as the frequency of gas-rich mergers as a function of redshift and environment, the star formation histories of the host galaxies, and their X-ray properties, for example.

Banfield et al. (2014) also studied the $1.4 \mathrm{GHz}$ polarization properties of a large sample of radio galaxies and radio-loud QSOs taken from the catalog presented in Hammond et al. (2012). Banfield et al. (2014) discussed the difference in the degree of polarization between radio galaxies and radio-loud QSOs as a result of a cosmic evolution in the space density of quiescent galaxies (i.e., the typical hosts of LERGs). They also found no significant difference in the RM distribution of the two types of sources but concluded that most of the extragalactic RM must originate close to the source. Such studies have important implications for predicting the number density of polarized sources that will be detected in deep polarization surveys with the SKA and its precursors/ pathfinders. The integrated degree of polarization of radioloud $\mathrm{AGN}$ at $1.4 \mathrm{GHz}$ has been claimed to be anticorrelated with the total radio flux density (e.g., Mesa et al. 2002; Tucci et al. 2004; Taylor et al. 2007; Subrahmanyan et al. 2010; Stil et al. 2014), and although this relation is disputed (Hales et al. 2014), it was claimed that it is the intrinsically less luminous radio sources that are more highly polarized (Banfield et al. 2011, 2014). Preliminary efforts were made to explain the anticorrelation based on a change in the 
dominant population of polarized radio sources from FR2s to FR1s toward lower flux densities (O'Sullivan et al. 2008; Stil et al. 2014). However, the results of this paper strongly suggest this is not the case. We also find a weak but significant anticorrelation between the $1.4 \mathrm{GHz}$ radio luminosity and $\Pi_{1.4 \mathrm{GHz}}$ (Section 3.2) and instead claim that it is most likely driven by the difference in $\Pi_{1.4 \mathrm{GHz}}$ between radio-loud radiative-mode $\mathrm{AGN}$, which dominate at high luminosities, and the jet-mode AGN, which dominate at lower luminosities.

\subsection{Jet Production Efficiency}

After likening the large-scale magnetoionic environment of radio sources to their host galaxy accretion states (i.e., HERGs and LERGs), it is interesting to discuss the potential implications of the accretion of magnetized gas from large distances in enabling the production of powerful, radioloud jets.

The existence of large amounts of poloidal magnetic flux close to a supermassive black hole is synonymous with jet production (e.g., McKinney \& Blandford 2009; Porth et al. 2011; Fendt et al. 2014) and also with the extraction of the rotational energy of the black hole in the so-called magnetically arrested accretion disk regime (MAD; Igumenshchev et al. 2003). In MAD systems, it is expected that the mass accretion rate and jet magnetic flux are strongly correlated (Tchekhovskoy et al. 2011), which is supported by recent observational results (Zamaninasab et al. 2014; Zdziarski et al. 2014). However, these observations are of sources with radiatively efficient accretion, and the MAD systems have only been successfully simulated in radiatively inefficient regimes. Furthermore, van Velzen \& Falcke (2013) have argued that the MAD model is inconsistent with the radiatively efficient accretion in radio-loud QSOs, on the grounds that the spread in the optical-radio luminosity relation does not allow for a large range of black hole spins. Mocz \& Guo (2015) presented a model for interpreting MADs in both the radiatively efficient and radiatively inefficient accretion regimes, but this is based on the assumption a MAD can be successfully formed in the radiatively efficient accretion regime.

In any case, the amounts of magnetic flux required to generate such strong poloidal fields close to the black hole most likely need to be advected from large distances, since the generation of such large magnetic flux in situ is considered unlikely (e.g., Begelman 2014). If the difference in the accretion mode of HERGs and LERGs is mainly due to the large-scale environment (i.e., HERGs have a radiatively efficient accretion flow due to a large supply of cold gas and LERGs have a radiatively inefficient accretion flow due to a hot interstellar medium), then the ability of the accretion flow to form a MAD system and produce powerful radio-loud jets may be related to the large-scale magnetized environment. Thus, the study of the large-scale magnetoionic environment of HERGs and LERGs through their polarization and Faraday rotation properties may provide important constraints on the likelihood of formation of a MAD.

In Section 3.5.3, we found that the sources with low Eddington-scaled accretion rates $\left(L_{\text {total }} / L_{\text {Edd }}<0.5 \%\right)$ could achieve higher values of $\Pi_{1.4 \mathrm{GHz}}$ than the sources with $L_{\text {total }} / L_{\text {Edd }}>0.5 \%$. This could be explained by a more uniform jet/lobe magnetic field structure and/or less Faraday depolarization in the environments of sources with $L_{\text {total }} / L_{\text {Edd }}<0.5 \%$. Our analysis of the effect of radio morphology on $\Pi_{1.4 \mathrm{GHz}}$ suggests that intrinsic magnetic field differences are not the dominant effect. We also find no significant difference between the RMs of strongly and weakly accreting sources, but we claim that the currently available RM data are dominated by the RM contribution from the Milky Way and thus insufficient to determine the true RM contribution local to the source. Higher precision RMs from broadband and high angular resolution radio observations should provide a robust test of this scenario through direct estimation of the amount of Faraday depolarization local to the source.

AGN that accrete in a radiatively efficient manner are considered to be the dominant type of AGN at high redshift and possibly responsible for the co-evolution of supermassive black holes and their host galaxies. Why only a small fraction $(\lesssim 10 \%)$ of these radiatively efficient AGN are radio-loud remains an open question. One proposed solution is the "magnetic flux paradigm" of Sikora \& Begelman (2013), who propose that the radiatively efficient radio-loud AGN must have undergone a previous radiatively inefficient accretion phase in order to build up the large amounts of magnetic flux required for powerful jet production (cf. Lubow et al. 1994). One way in which this could be achieved is by a major merger between a disk galaxy and a giant elliptical galaxy that has been undergoing hot accretion for some time. Such a scenario has some observational support in that the most luminous AGN are often triggered by mergers (e.g., Ramos Almeida et al. 2012). Whether or not giant elliptical galaxies have sufficient amounts of coherent magnetic fields that can be dragged inwards to the black hole horizon is currently unclear; however, this is not inconsistent with our observations of highly polarized LERGs whose elliptical galaxy hosts would cause smaller amounts of Faraday depolarization if large-scale coherent magnetic fields were indeed present. Upcoming broadband radio polarization data from surveys, such as POSSUM on the ASKAP telescope (Gaensler et al. 2010) and the proposed VLA Sky Survey, can provide important constraints on this proposed scenario.

\section{CONCLUSIONS}

We have presented a radio polarization study of 796 radioloud AGN $(z<0.7)$ in relation to their host galaxy properties, accretion states and large-scale environments. The integrated degree of polarization at $1.4 \mathrm{GHz}$ was obtained from the NVSS in the region that overlapped with the SDSS survey area. We used the SDSS optical spectroscopic classifications of Best \& Heckman (2012), who categorized a large sample of NVSS radio sources into high-excitation and low-excitation radio galaxies (i.e., HERGs and LERGs). We find a fundamental difference in the polarization properties between HERGs and LERGs, where the LERGs are observed to span the full range of expected integrated degrees of polarization at $1.4 \mathrm{GHz}$ $\left(\Pi_{1.4 \mathrm{GHz}}\right)$, with a maximum of $\sim 30 \%$, while HERGs (and radio-loud QSOs) are restricted to a maximum of $\Pi_{1.4 \mathrm{GHz}} \sim 15 \%$. We also find that the weakly accreting LERG sources (with Eddington ratios $<0.5 \%$ ) can attain higher values of $\Pi_{1.4 \mathrm{GHz}}$ than the strongly accreting LERGs and HERGs (with Eddington ratios $>0.5 \%$ ). This may be important for attempts to explain the production of powerful radio-loud jets by the ability of AGN host galaxies to accumulate large amounts of magnetic flux close to the black hole (e.g., Sikora \& Begelman 2013). Infrared data from WISE allowed us to determine the host galaxy type, with LERGs mostly inhabiting 
early-type elliptical galaxies and HERGs typically with diskdominated hosts. This is broadly consistent with detailed optical studies which find that the host galaxies of HERGs are typically of lower stellar mass and have bluer colors than LERG hosts (e.g., Heckman \& Best 2014). Thus, we maintain that the results from our sample of polarized HERGs and LERGs are applicable to the HERG and LERG populations in general.

The scenario that we consider to best explain the current data is one in which the full range of polarization, from $<1 \%$ to $\sim 30 \%$ of the LERGs, can be explained by the full range of environments in which LERGs are observed to reside, in the local universe. In high gas density environments, the jet emission structure is more likely to be bent, causing polarization angle cancellation, while significant Faraday depolarization caused by the high-density magnetoionic medium can help to further suppress the observed integrated polarization at $1.4 \mathrm{GHz}$. In poor group environments, the jets are more likely to remain straight, and along with the lower expected amount of external Faraday depolarization, this leads to the highest integrated degree of polarization. Additionally, the LERG sources with $\Pi_{1.4 \mathrm{GHz}}>15 \%$ have significantly smaller linear sizes than the other LERGs, potentially indicating that these LERGs may also have intrinsically weaker jets. The restricted integrated degree of polarization in HERGs is not easily explained since the HERGs reside in a lower galaxy density environment, on average, than the LERGs. However, we suggest that the high ionizing luminosity of the central engine ionizes a significant fraction of the host galaxy, increasing the amount of magnetoionic material contributing to the Faraday rotation local to the source. This would be expected to generate large amounts of Faraday depolarization in the HERG environment, potentially restricting the integrated degree of polarization at $1.4 \mathrm{GHz}$ from reaching values greater than $\sim 15 \%$.

However, although we expect Faraday effects to dominate at 1.4 GHz, we do not find any direct evidence for this from the Faraday RMs derived from the NVSS data in Taylor et al. (2009). These RMs have been shown to be dominated by the magnetoionic material of the Milky Way and are relatively insensitive probes of the local environments of extragalactic radio sources (e.g., Oppermann et al. 2015); thus, we cannot test our above hypothesis in a robust manner. We have also considered intrinsic magnetic field differences of the largescale radio morphological classes (i.e., FR1, FR2, and FR0) as a potential origin of the difference in the polarization properties of the HERGs and LERGs. However, we do not find any conclusive evidence suggesting that the FR morphological class is the primary driver. To understand the relative importance of Faraday rotation and intrinsic magnetic field structure in determining the polarization properties of HERGs and LERGs requires high-precision RM and polarization measurement across a wide range of frequencies. Upcoming broadband radio polarization surveys, such as the Polarization Sky Survey of the Universe's Magnetism (POSSUM) on the Australian Square Kilometre Array (SKA) Pathfinder telescope (ASKAP) in conjunction with the proposed VLA Sky Survey, will enable much more robust inferences on the intrinsic magnetic field and magnetoionic environment of HERG and LERG sources.
S.P.O.'S and B.M.G. acknowledge the support of the Australian Research Council through grants FS100100033 and FL100100114, respectively. The authors would like to thank Jeroen Stil for advice on the polarization bias correction and the referee for several important comments which significantly improved the paper. This research has made use of NASA's Astrophysics Data System Service and the NASA/ IPAC Extragalactic Database (NED) that is operated by the Jet Propulsion Laboratory, California Institute of Technology, under contract with the National Aeronautics and Space Administration. This research made use of TOPCAT, an interactive graphical viewer and editor for tabular data (Taylor 2005).

\section{APPENDIX \\ THE BRIGHTEST RADIO SOURCES: $I>100 \mathrm{mJy}$}

Stil et al. (2014) showed that the median degree of polarization of all NVSS sources increases toward lower flux densities (e.g., the presented fit to their data gives a median degree of polarization of $1.93 \%$ at $100 \mathrm{mJy}$ and $2.17 \%$ at 10 mJy). Therefore, we split our sample at $I=100 \mathrm{mJy}$ in order to compare the properties of the brighter sources to the fainter sources in our sample. At $I>100 \mathrm{mJy}$, there are 211 LERGs and 37 HERGs. We find no significant difference in $\Pi_{1.4 \mathrm{GHz}}$ between HERGs and LERGs for $I>100 \mathrm{mJy}$. The number of LERGs in our sample with $\Pi_{1.4 \mathrm{GHz}}>15 \%$ decreases from 71 at $I<100 \mathrm{mJy}(\sim 10 \%$ of LERGs $)$ to only 2 at $I>100 \mathrm{mJy}$ ( $\sim 1 \%$ of LERGs).

In Figure 17, we repeat Figures 4, 6, 7, and 8 from the main text but now only include sources with $I>100 \mathrm{mJy}$. Comparing Figure 17(a) to Figure 4, we see that there is no strong redshift dependence on flux in our sample. The median redshift changes from 0.18 to 0.16 for LERGs, and from 0.25 to 0.23 for HERGs. The majority $(88 \%)$ of the radio-loud QSOs have $I>100 \mathrm{mJy}$. In Figure $17(\mathrm{~b})$ and (c), we see that most sources with $L_{1.4 \mathrm{GHz}}<10^{24} \mathrm{~W} \mathrm{~Hz}^{-1}$ are removed. In fact, the median radio luminosity increases by a factor of approximately 2 for both HERGs and LERGs and there is no longer any significant evidence for a correlation between $\Pi_{1.4 \mathrm{GHz}}$ and $L_{1.4 \mathrm{GHz}}$. Figure $17(\mathrm{~d})$ confirms that, even for the brightest sources, the radio morphology does not show any clear split in radio luminosity into FR1- and FR2-type sources. There is no significant difference in $\Pi_{1.4 \mathrm{GHz}}$ between FR1, FR2, or FR0 source morphologies.

Interestingly, the median total intensity spectral index of sources with $I>100 \mathrm{mJy}$ is -0.7 while it is -0.6 for $I<100$ mJy. If the spectral index is a useful probe of the environment density (as argued in Section 3.4.4), then the less steep spectral index for sources with $I<100 \mathrm{mJy}$ may explain why there is a greater fraction of LERGs with $\Pi_{1.4 \mathrm{GHz}}>15 \%$ at these lower fluxes. This provides some support for our conclusion that a sub-population of polarized LERGs with weaker jets and smaller sizes in underdense environments can achieve $\Pi_{1.4 \mathrm{GHz}}>15 \%$. We have spectral index measurements for $\sim 60 \%$ of sources with $I>100 \mathrm{mJy}$, but for only $\sim 20 \%$ of sources with $I<100 \mathrm{mJy}$. The upcoming broadband radio polarization surveys will provide much better statistics in order to investigate this in more detail. 


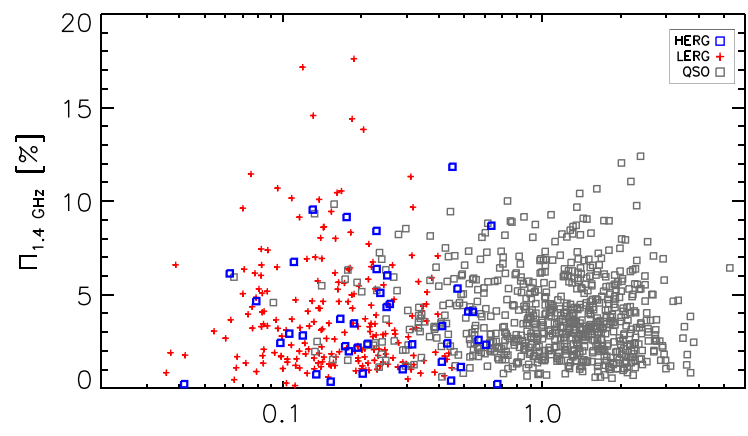

(a)

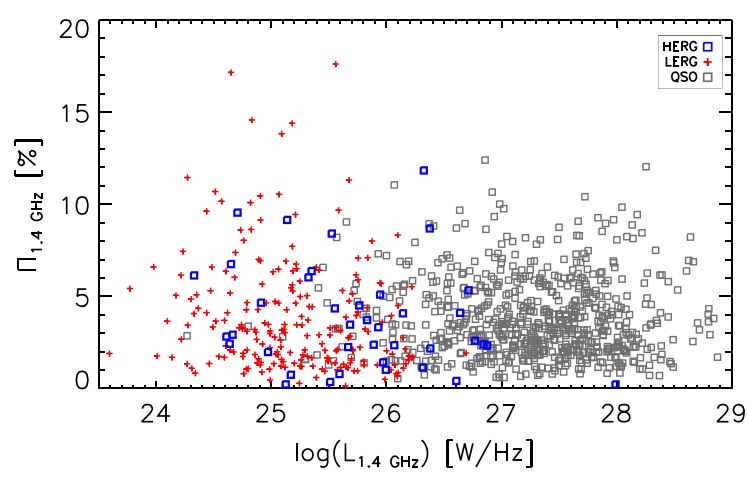

(c)

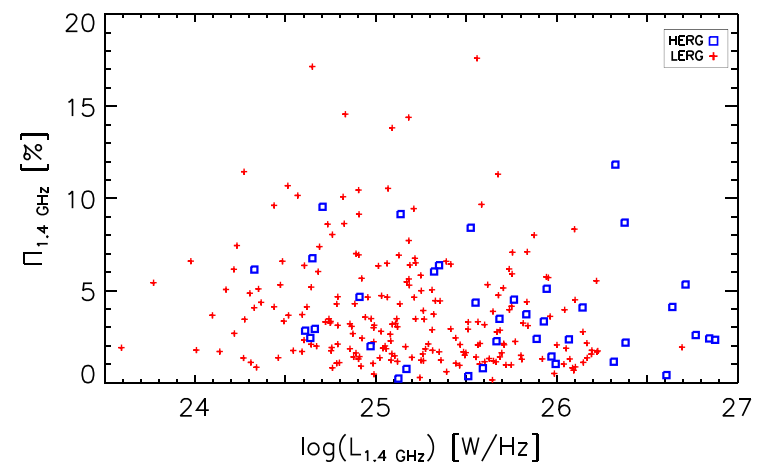

(b)

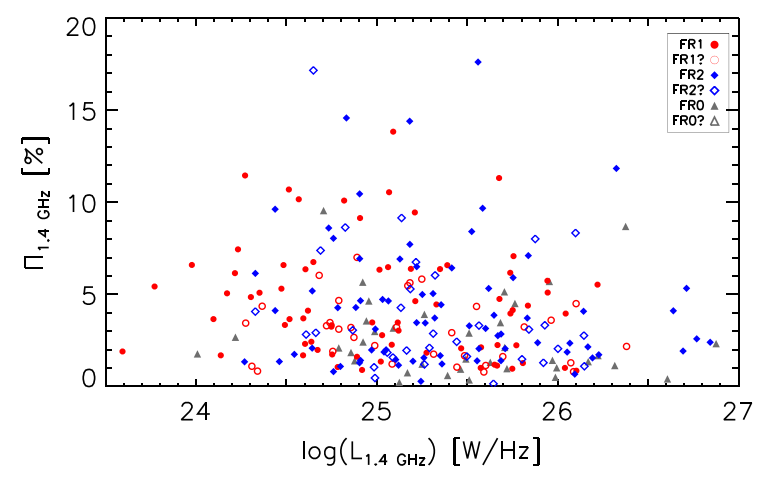

(d)

Figure 17. Parts (a), (b), (c), and (d) correspond to Figures 4, 6, 7, and 8 of the main text, respectively, but now only including sources with total radio flux density greater than $100 \mathrm{mJy}$.

\section{REFERENCES}

Abazajian, K. N., Adelman-McCarthy, J. K., Agüeros, M. A., et al. 2009, ApJS, 182, 543

Ade, P. A. R., Aghanim, N., et al. Planck Collaboration 2014, A\&A, 571, A16 Antweiler, R., \& Taylor, H. 2008, EnST, 42, 3732

Baldi, R. D., Capetti, A., \& Giovannini, G. 2015, A\&A, 576, A38

Baldwin, J. E., \& Scott, P. F. 1973, MNRAS, 165, 259

Banfield, J. K., George, S. J., Taylor, A. R., et al. 2011, ApJ, 733, 69

Banfield, J. K., Schnitzeler, D. H. F. M., George, S. J., et al. 2014, MNRAS, 444, 700

Baum, S. A., \& Heckman, T. 1989, ApJ, 336, 702

Becker, R. H., White, R. L., \& Helfand, D. J. 1995, ApJ, 450, 559

Begelman, M. C. 2014, in Proc. 26th Solvay Conf. Physics: "Astrophysics and Cosmology”, ed. R. Blandford \& A. Sevrin (Singapore: World Scientific), in press (arXiv:1410.8132)

Bernardi, M., Sheth, R. K., Annis, J., et al. 2003, AJ, 125, 1849

Best, P. N. 2004, MNRAS, 351, 70

Best, P. N. 2009, AN, 330, 184

Best, P. N., \& Heckman, T. M. 2012, MNRAS, 421, 1569

Best, P. N., Kaiser, C. R., Heckman, T. M., \& Kauffmann, G. 2006, MNRAS, 368, L67

Best, P. N., Kauffmann, G., Heckman, T. M., et al. 2005, MNRAS, 362, 25

Bîrzan, L., McNamara, B. R., Nulsen, P. E. J., Carilli, C. L., \& Wise, M. W. 2008, ApJ, 686, 859

Blandford, R. D., \& Payne, D. G. 1982, MNRAS, 199, 883

Blandford, R. D., \& Znajek, R. L. 1977, MNRAS, 179, 433

Brinchmann, J., Charlot, S., Heckman, T. M., et al. 2004, arXiv:astro-ph/ 0406220

Buttiglione, S., Capetti, A., Celotti, A., et al. 2010, A\&A, 509, A6

Cavagnolo, K. W., McNamara, B. R., Nulsen, P. E. J., et al. 2010, ApJ, 720, 1066

Condon, J. J., Cotton, W. D., Greisen, E. W., et al. 1998, AJ, 115, 1693

Croton, D. J., Springel, V., White, S. D. M., et al. 2006, MNRAS, 365, 11

de Gasperin, F., Merloni, A., Sell, P., et al. 2011, MNRAS, 415, 2910
Fanaroff, B. L., \& Riley, J. M. 1974, MNRAS, 167, 31P

Farnes, J. S., Gaensler, B. M., \& Carretti, E. 2014, ApJS, 212, 15

Fendt, C., Porth, O., \& Sheikhnezami, S. 2014, IJMPS, 28, 60190

Gaensler, B. M., Landecker, T. L., Taylor, A. R., \& POSSUM Collaboration 2010, BAAS, 42, 47013

Garrington, S. T., Leahy, J. P., Conway, R. G., \& Laing, R. A. 1988, Natur, 331,147

Gendre, M. A., Best, P. N., Wall, J. V., \& Ker, L. M. 2013, MNRAS, 430, 3086

Godfrey, L. E. H., \& Shabala, S. S. 2013, ApJ, 767, 12

Goodlet, J. A., \& Kaiser, C. R. 2005, MNRAS, 359, 1456

Grant, J. K. 2011, PhD thesis, Univ. Calgary

Guidetti, D., Laing, R. A., Croston, J. H., Bridle, A. H., \& Parma, P. 2012, MNRAS, 423, 1335

Gürkan, G., Hardcastle, M. J., \& Jarvis, M. J. 2014, MNRAS, 438, 1149

Hainline, K. N., Hickox, R. C., Greene, J. E., et al. 2014, ApJ, 787, 65

Hales, C. A., Norris, R. P., Gaensler, B. M., \& Middelberg, E. 2014, MNRAS, 440,3113

Hammond, A. M., Robishaw, T., \& Gaensler, B. M. 2012, arXiv:1209.1438v3

Hancock, P. J., Murphy, T., Gaensler, B. M., Hopkins, A., \& Curran, J. R. 2012, MNRAS, 422, 1812

Hardcastle, M. J. 2004, A\&A, 414, 927

Hardcastle, M. J., Evans, D. A., \& Croston, J. H. 2007, MNRAS, 376, 1849

Hardcastle, M. J., \& Krause, M. G. H. 2014, MNRAS, 443, 1482

Heckman, T. M., \& Best, P. N. 2014, ARA\&A, 52, 589

Heckman, T. M., Kauffmann, G., Brinchmann, J., et al. 2004, ApJ, 613, 109

Hine, R. G., \& Longair, M. S. 1979, MNRAS, 188, 111

Igumenshchev, I. V., Narayan, R., \& Abramowicz, M. A. 2003, ApJ, 592, 1042

Ineson, J., Croston, J. H., Hardcastle, M. J., et al. 2013, ApJ, 770, 136

Kauffmann, G., \& Heckman, T. M. 2009, MNRAS, 397, 135

Klamer, I. J., Ekers, R. D., Bryant, J. J., et al. 2006, MNRAS, 371, 852

Laing, R. A. 1981, ApJ, 248, 87

Laing, R. A. 1988, Natur, 331, 149

Laing, R. A., \& Bridle, A. H. 2014, MNRAS, 437, 3405

Laing, R. A., Bridle, A. H., Parma, P., \& Murgia, M. 2008, MNRAS, 391, 521 
Laing, R. A., Jenkins, C. R., Wall, J. V., \& Unger, S. W. 1994, in ASP Conf. Ser. 54, The First Stromlo Symposium: The Physics of Active Galaxies, ed. G. V. Bicknell, M. A. Dopita \& P. J. Quinn (San Francisco, CA: ASP), 201 Lara-López, M. A., Cepa, J., Bongiovanni, A., et al. 2009, A\&A, 505, 529 Ledlow, M. J., \& Owen, F. N. 1996, AJ, 112, 9

Lin, Y.-T., Shen, Y., Strauss, M. A., Richards, G. T., \& Lunnan, R. 2010, ApJ, 723, 1119

Lubow, S. H., Papaloizou, J. C. B., \& Pringle, J. E. 1994, MNRAS, 267, 235

McCarthy, P. J., van Breugel, W., Spinrad, H., \& Djorgovski, S. 1987, ApJL, 321, L29

McKinney, J. C., \& Blandford, R. D. 2009, MNRAS, 394, L126

Meier, D. L. 2002, NewAR, 46, 247

Mesa, D., Baccigalupi, C., de Zotti, G., et al. 2002, A\&A, 396, 463

Mingo, B., Hardcastle, M. J., Croston, J. H., et al. 2014, MNRAS, 440, 269

Mocz, P., \& Guo, X. 2015, MNRAS, 447, 1498

Narayan, R., \& Yi, I. 1995, ApJ, 452, 710

Netzer, H., Mainieri, V., Rosati, P., \& Trakhtenbrot, B. 2006, A\&A, 453, 525

Oppermann, N., Junklewitz, H., Greiner, M., et al. 2015, A\&A, 575, A118

O'Sullivan, S., Stil, J., Taylor, A. R., et al. 2008, in Proc. 9th European VLBI Network Symp., The Role of VLBI in the Golden Age for Radio Astronomy, http://pos.sissa.it/cgi-bin/reader/conf.cgi?confid=72, 107

Owen, F. N., \& Rudnick, L. 1976, ApJL, 205, L1

Pacholczyk, A. G. 1970, Radio Astrophysics (San Francisco: Freeman)

Pedelty, J. A., Rudnick, L., McCarthy, P. J., \& Spinrad, H. 1989, AJ, 97, 647

Porth, O., Fendt, C., Meliani, Z., \& Vaidya, B. 2011, ApJ, 737, 42

Ramos Almeida, C., Bessiere, P. S., Tadhunter, C. N., et al. 2012, MNRAS, 419, 687

Rawlings, S., \& Saunders, R. 1991, Natur, 349, 138

Runnoe, J. C., Brotherton, M. S., \& Shang, Z. 2012, MNRAS, 426, 2677

Sadler, E. M., Ekers, R. D., Mahony, E. K., Mauch, T., \& Murphy, T. 2014, MNRAS, 438, 796

Saikia, D. J., \& Salter, C. J. 1988, ARA\&A, 26, 93

Schnitzeler, D. H. F. M. 2010, MNRAS, 409, L99
Shakura, N. I., \& Sunyaev, R. A. 1973, A\&A, 24, 337

Shi, H., Liang, H., Han, J. L., \& Hunstead, R. W. 2010, MNRAS, 409, 821

Shimasaku, K., Fukugita, M., Doi, M., et al. 2001, AJ, 122, 1238

Sikora, M., \& Begelman, M. C. 2013, ApJL, 764, L24

Simmons, J. F. L., \& Stewart, B. G. 1985, A\&A, 142, 100

Slee, O. B., Siegman, C. B., \& Wilson, I. R. G. 1983, AuJPh, 36, 101

Stawarz, L., Sikora, M., \& Lasota, J.-P. 2008, in ASP Conf. Ser. 386, Extragalactic Jets: Theory and Observation from Radio to Gamma Ray, Rector, T. A., \& De Young, D. S. (San Francisco, CA: ASP), 169

Stil, J. M., Keller, B. W., George, S. J., \& Taylor, A. R. 2014, ApJ, 787, 99

Stil, J. M., Taylor, A. R., \& Sunstrum, C. 2011, ApJ, 726, 4

Subrahmanyan, R., Ekers, R. D., Saripalli, L., \& Sadler, E. M. 2010, MNRAS, 402, 2792

Taylor, A. R., Stil, J. M., \& Sunstrum, C. 2009, ApJ, 702, 1230

Taylor, G. B., Fabian, A. C., Gentile, G., et al. 2007, MNRAS, 382, 67

Taylor, M. B. 2005, Software and Systems XIV, in ASP Conf. Ser. 347, Astronomical Data Analysis, Shopbell, P., Britton, M., \& Ebert, R. (San Francisco, CA: ASP), 29

Tchekhovskoy, A., McKinney, J. C., \& Narayan, R. 2012, JPhCS, 372, 012040

Tchekhovskoy, A., Narayan, R., \& McKinney, J. C. 2011, MNRAS, 418, L79

Tremaine, S., Gebhardt, K., Bender, R., et al. 2002, ApJ, 574, 740

Tucci, M., Martínez-González, E., Toffolatti, L., González-Nuevo, J., \& de Zotti, G. 2004, MNRAS, 349, 1267

Urry, C. M., \& Padovani, P. 1995, PASP, 107, 803

van Velzen, S., \& Falcke, H. 2013, A\&A, 557, L7

van Velzen, S., Falcke, H., \& Körding, E. 2015, MNRAS, 446, 2985

Wing, J. D., \& Blanton, E. L. 2011, AJ, 141, 88

Wright, E. L., Eisenhardt, P. R. M., Mainzer, A. K., et al. 2010, AJ, 140, 1868

Zamaninasab, M., Clausen-Brown, E., Savolainen, T., \& Tchekhovskoy, A. 2014, Natur, 510, 126

Zdziarski, A. A., Sikora, M., Pjanka, P., \& Tchekhovskoy, A. 2014, arXiv: 1410.7310 